\title{
Pacific
}

Journal of

Mathematics

\section{THE SU(N) CASSON-LIN INVARIANTS FOR LINKS}

HANS U. BODEN AND ERIC HARPER 


\title{
THE SU $(N)$ CASSON-LIN INVARIANTS FOR LINKS
}

\author{
HANS U. BODEN AND ERIC HARPER
}

\begin{abstract}
We introduce the $\mathrm{SU}(N)$ Casson-Lin invariants for links $L$ in $S^{3}$ with more than one component. Writing $L=\ell_{1} \cup \cdots \cup \ell_{n}$, we require as input an $n$-tuple $\left(a_{1}, \ldots, a_{n}\right) \in \mathbb{Z}^{n}$ of labels, where $a_{j}$ is associated with $\ell_{j}$. The $\mathrm{SU}(N)$ Casson-Lin invariant, denoted $h_{N, a}(L)$, gives an algebraic count of certain projective $\mathrm{SU}(N)$ representations of the link group $\pi_{1}\left(S^{3} \backslash L\right)$, and the family $h_{N, a}$ of link invariants gives a natural extension of the $\mathrm{SU}(2)$ Casson-Lin invariant, which was defined for knots by X.-S. Lin and for 2-component links by Harper and Saveliev. We compute the invariants for the Hopf link and more generally for chain links, and we show that, under mild conditions on the labels $\left(a_{1}, \ldots, a_{n}\right)$, the invariants $h_{N, a}(L)$ vanish whenever $L$ is a split link.
\end{abstract}

\section{Introduction}

The goal of this paper is to construct $\mathrm{SU}(N)$ Casson-Lin invariants $h_{N, a}(L)$ for oriented links $L$ in $S^{3}$. These invariants are defined as a signed count of conjugacy classes of certain irreducible projective $\mathrm{SU}(N)$ representations of $\pi_{1}\left(S^{3} \backslash L\right)$ with a nontrivial 2-cocycle. Given an oriented link $L$ with $n$ components, the 2-cocycle is determined by an $n$-tuple $a=\left(a_{1}, \ldots, a_{n}\right) \in \mathbb{Z}^{n}$ of labels, and the choice of labels is made so that the resulting 2-cocycle is nontrivial. This is critical in what follows because it prohibits the existence of reducibles; see Proposition 2.2. We denote the resulting algebraic count as $h_{N, a}(L)$, and the following theorem is the main result of this paper.

Main theorem. Suppose $L \subset S^{3}$ is an oriented $n$-component link with $n \geq 2$ and $a=\left(a_{1}, \ldots, a_{n}\right)$ is an allowable $n$-tuple of labels. Then the integer $h_{N, a}(L)$ is a well-defined invariant of $L$.

We briefly outline how the above theorem is established. By Alexander's theorem [1923], every link $L \subset S^{3}$ can be realized as the closure $L=\hat{\sigma}$ for some braid $\sigma \in B_{k}$.

Boden was supported by a grant from the Natural Sciences and Engineering Research Council of Canada. Harper was supported by CIRGET and McMaster postdoctoral fellowships.

MSC2010: 20C15, 57M25.

Keywords: braids, links, representation spaces, Casson-Lin invariant. 
The braid group $B_{k}$ acts naturally on the free group $F_{k}$, and this induces an action on the space of $\mathrm{SU}(N)$ representations of $F_{k}$, which we denote as

$$
R_{k}=\operatorname{Hom}\left(F_{k}, \mathrm{SU}(N)\right)=\mathrm{SU}(N) \times \cdots \times \mathrm{SU}(N) .
$$

We extend this action to the wreath product $\mathbb{Z}_{N} 2 B_{k}$ as follows. Identifying $\mathbb{Z}_{N}$ with the center of $\mathrm{SU}(N)$, for $\varepsilon=\left(\varepsilon_{1}, \ldots, \varepsilon_{k}\right) \in\left(\mathbb{Z}_{N}\right)^{k}$ and $X=\left(X_{1}, \ldots, X_{k}\right) \in R_{k}$, we set $(\varepsilon, \sigma)(X)=\left(\varepsilon_{1} \sigma(X)_{1}, \ldots, \varepsilon_{k} \sigma(X)_{k}\right)$. This extends the braid group action on $R_{k}$ to an action of $\mathbb{Z}_{N} 2 B_{k}$, and in fact every fixed point Fix $(\varepsilon \sigma) \subseteq R_{k}$ can be identified with a projective representation of the link group $G_{L}=\pi_{1}\left(S^{3} \backslash L\right)$.

The key result is Proposition 2.2, which shows that every element $X \in \operatorname{Fix}(\varepsilon \sigma)$ is irreducible. Consequently, writing $R_{k}^{*} \subset R_{k}$ for the subspace of irreducible $\mathrm{SU}(N)$ representations, Proposition 2.2 implies that the graph $\Gamma_{\varepsilon \sigma}^{*}$ and the diagonal $\Delta_{k}^{*}$ intersect in a compact subset of $R_{k}^{*} \times R_{k}^{*}$. It follows that one can arrange transversality of the intersection $\Gamma_{\varepsilon \sigma}^{*} \cap \Delta_{k}^{*}$ by a compactly supported isotopy, and using natural orientations on the quotients $\hat{\Gamma}_{\varepsilon \sigma}=\Gamma_{\varepsilon \sigma}^{*} / \mathrm{PU}(N)$ and $\hat{\Delta}_{k}=\Delta_{k}^{*} / \mathrm{PU}(N)$, we define $h_{N, a}(\varepsilon \sigma)$ as the oriented intersection number of $\hat{\Gamma}_{\varepsilon \sigma}$ and $\hat{\Delta}_{k}$. Our main result is then established by showing that $h_{N, a}(\varepsilon \sigma)$ is independent of the choice of compatible $k$-tuple $\varepsilon=\left(\varepsilon_{1}, \ldots, \varepsilon_{k}\right)$ (Proposition 3.4), and that it is invariant under the two Markov moves (Propositions 3.5 and 3.6). It follows that $h_{N, a}(L)$ gives a well-defined invariant of the link $L \subset S^{3}$.

One of the virtues of this approach is that it leads to a direct method for computing the invariants, and we illustrate this by computing $h_{N, a}(L)$ for the Hopf link and for chain links (Propositions 4.3 and 4.5) and by showing that the invariants vanish for split links (Proposition 4.6).

Gauge Theory. One motivation for defining link invariants in terms of the $\mathrm{SU}(N)$ representations of the link group is that these representations can be identified with flat connections on a principal $\mathrm{SU}(N)$ bundle over the link exterior, which allows for a gauge theoretic interpretation. This approach was originally used by Taubes [1990] to interpret Casson's invariant $\lambda(\Sigma)$ of homology 3-spheres $\Sigma$ in terms of flat SU(2) connections, and using similar ideas, Floer [1988] defined $\mathbb{Z}_{8}$-graded groups $\mathrm{HF}_{*}(\Sigma)$ called the instanton Floer homology and whose Euler characteristic equals the Casson invariant.

The Casson-Lin invariants can also be interpreted gauge theoretically, as we now explain. X.-S. Lin [1992] originally defined the invariant $h(K)$ of knots $K \subset S^{3}$ as an algebraic count of conjugacy classes of tracefree irreducible $\mathrm{SU}(2)$ representations of $\pi_{1}\left(S^{3} \backslash K\right)$ and proved that $h(K)=\operatorname{sign}(K) / 2$, half the signature of $K$. More recently, C. Herald [1997] used gauge theory to define an extended Casson-Lin invariant $h_{\alpha}(K)$ for knots $K \subset \Sigma^{3}$ in homology 3-spheres which allows for more general meridional trace conditions, and he generalized Lin's formula by 
showing that $h_{\alpha}(K)=\operatorname{sign}_{\alpha}(K) / 2$, half the Tristram-Levine $\alpha$-twisted signature of $K$. (Similar results were obtained by M. Heusener and J. Kroll [1998].) O. Collin and B. Steer [1999] then used moduli spaces of orbifold connections to define an associated Floer homology theory for knots whose Euler characteristic equals $h_{\alpha}(K)$ and P. Kronheimer and T. Mrowka [2011b] further developed the instanton Floer homology theory of knots in, and they used it to prove a strong nontriviality result for Khovanov homology [Kronheimer and Mrowka 2011a].

Harper and N. Saveliev [2010] used projective SU(2) representations to extend the Casson-Lin invariant to 2-component links $L$ in $S^{3}$, and they showed that $h(L)= \pm \operatorname{lk}\left(\ell_{1}, \ell_{2}\right)$, where $\operatorname{lk}\left(\ell_{1}, \ell_{2}\right)$ is the linking number of $L=\ell_{1} \cup \ell_{2}$. They gave a gauge theoretic description of the invariant $h(L)$ in [2012], where they also described Floer homology groups with Euler characteristic equal to $h(L)$.

In view of all of these results, it is natural to ask whether the $\mathrm{SU}(N)$ Casson-Lin invariants introduced here can also be interpreted gauge theoretically. We plan to address this question in a future article using moduli spaces of projective $\mathrm{SU}(N)$ representations; see [Ruberman and Saveliev 2004]. We hope to use this approach to extend the Casson-Lin invariants $h_{N, a}(L)$ to links $L \subset \Sigma^{3}$ in homology 3-spheres and to describe corresponding Floer homology groups. In particular, we expect this approach will help clarify the relationship between the invariants $h_{N, a}(L)$ studied here and the $\mathrm{SU}(N)$ instanton Floer groups constructed by Kronheimer and Mrowka [2011b]. It is possible that this approach will also shed light on other interesting questions, such as whether and how the Casson-Lin invariants are related to classical link invariants, such as the higher linking numbers.

We give a brief outline of the contents of this paper. In Section 1, we introduce the notation for braids $\sigma \in B_{k}$, links $L \subset S^{3}$, and $\mathrm{SU}(N)$ representations that is used throughout the article. In Section 2, we introduce allowable labels $\left(a_{1}, \ldots, a_{n}\right)$ for a given $n$-component link $L \subset S^{3}$, and a compatible $k$-tuple $\left(\varepsilon_{1}, \ldots, \varepsilon_{k}\right)$ for a braid $\sigma \in B_{k}$ with closure $L$. We also introduce projective $\mathrm{SU}(N)$ representations of the link group $G_{L}$ and establish irreducibility of elements of Fix $(\varepsilon \sigma)$. In Section 3, we define the invariant $h_{N, a}(L)$ as an oriented intersection number and prove it is independent of the various choices involved. In Section 4, we calculate the invariants $h_{N, a}(L)$ for the Hopf link and the $n$-component chain link, and we prove a general vanishing result for the invariants for split links.

\section{Braids and representations}

In this section, we introduce the results for braids, links, and $\mathrm{SU}(N)$ representations that will be used throughout the article.

1A. The braid group. We denote by $B_{k}$ the group of geometric braids on $k$ strands with standard generators $\sigma_{1}, \ldots, \sigma_{k-1}$ and relations $\sigma_{i} \sigma_{j}=\sigma_{j} \sigma_{i}$ for $|j-i|>1$ and 


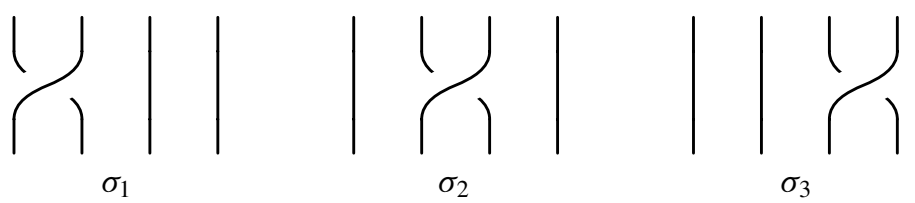

Figure 1. The three generators of $B_{4}$.

$\sigma_{i} \sigma_{i+1} \sigma_{i}=\sigma_{i+1} \sigma_{i} \sigma_{i+1}$. The generators are depicted in Figure 1. Note that in this paper we follow Convention 1.13 of [Kassel and Turaev 2008].

Each braid $\sigma \in B_{k}$ determines a permutation, and the resulting map $B_{k} \rightarrow S_{k}$, which sends the generator $\sigma_{i}$ to the transposition $\overline{\sigma_{i}}=(i, i+1)$, is a surjection. Given $\sigma \in B_{k}$, we let $\bar{\sigma} \in S_{k}$ denote the corresponding permutation. Under this map, the symmetric group $S_{k}$ acts on the set $\{1, \ldots, k\}$ on the right. For $i \in\{1, \ldots, k\}$, we write $(i)^{\bar{\sigma}}$ for the image of $i$ under $\bar{\sigma} \in S_{k}$.

Let $F_{k}$ be the free group with free generating set $x_{1}, \ldots, x_{k}$. There is a natural right action of $B_{k}$ on $F_{k}$ defined by setting $\sigma_{i}: F_{k} \rightarrow F_{k}$ to be the map

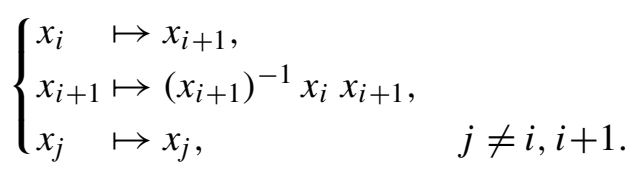

This action defines a faithful representation $\varrho: B_{k} \rightarrow \operatorname{Aut}\left(F_{k}\right)$, and we use it to identify $B_{k}$ with its image in $\operatorname{Aut}\left(F_{k}\right)$ under $\varrho$. As this is a right action, we will use $x_{i}^{\sigma}$ to denote the image of $x_{i}$ under $\sigma \in B_{k}$.

Example 1.1. We explain how to read the action of a braid, which is explained in [Fenn et al. 1997, Section 2.4] for the left action, and we present the details for the right action.

The basic idea is to view the free group $F_{k}$ as the fundamental group of a 2-disk with $k$ punctures and keep track of basepoints as you move the disk vertically, letting the punctures move along the braid. Specifically, label the top strands $x_{1}, \ldots, x_{k}$ from left to right. Then push the labels down, inserting a Wirtinger relation at each crossing. At the bottom of the braid the strands will be labeled by words $w_{1}, \ldots, w_{k}$ in $x_{1}, \ldots, x_{k}$, and the right action of $\sigma$ is given by the automorphism sending $x_{i}$ to $x_{i}^{\sigma}:=w_{i}$.

Using Figure 2, we determine the actions of $\sigma_{1} \sigma_{2}$ and $\sigma_{2} \sigma_{1}$ on $F_{3}=\left\langle x_{1}, x_{2}, x_{3}\right\rangle$ to be given by

$$
\left\{\begin{array} { l } 
{ x _ { 1 } ^ { \sigma _ { 1 } \sigma _ { 2 } } = x _ { 2 } , } \\
{ x _ { 2 } ^ { \sigma _ { 1 } \sigma _ { 2 } } = x _ { 3 } , } \\
{ x _ { 3 } ^ { \sigma _ { 1 } \sigma _ { 2 } } = x _ { 3 } ^ { - 1 } x _ { 2 } ^ { - 1 } x _ { 1 } x _ { 2 } x _ { 3 } , }
\end{array} \quad \text { and } \quad \left\{\begin{array}{l}
x_{1}^{\sigma_{2} \sigma_{1}}=x_{3}, \\
x_{2}^{\sigma_{2} \sigma_{1}}=x_{3}^{-1} x_{1} x_{3}, \\
x_{3}^{\sigma_{2} \sigma_{1}}=x_{3}^{-1} x_{2} x_{3} .
\end{array}\right.\right.
$$

We point out two facts about the action of $B_{k}$ on $F_{k}$, both of which are easily verified for each generator. Firstly, for any $\sigma \in B_{k}$, the permutation $\sigma$ acts by 

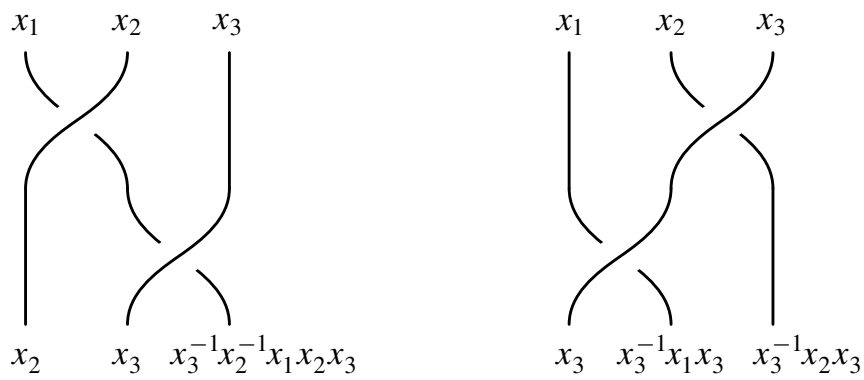

Figure 2. Reading the action of the braids $\sigma_{1} \sigma_{2}$ (left) and $\sigma_{2} \sigma_{1}$ (right).

conjugation and permutation on the generating set $x_{1}, \ldots, x_{k}$ for $F_{k}$. Indeed,

$$
x_{i}^{\sigma}=w x_{(i)^{\bar{\sigma}}} w^{-1},
$$

where $w \in F_{k}$ is some word depending on $\sigma$ and $i$. Secondly, every braid $\sigma \in B_{k}$ preserves the product $x_{1} \cdots x_{k}$,

$$
\left(x_{1} \cdots x_{k}\right)^{\sigma}=x_{1} \cdots x_{k} .
$$

1B. The group of a link. Every link $L$ in $S^{3}$ can be realized as the closure $L=\hat{\sigma}$ of a braid $\sigma$. We regard $L$ as an oriented link, where the strands of the braid $\sigma$ are oriented in the downward direction. The link group $G_{L}=\pi_{1}\left(S^{3} \backslash L\right)$ admits a standard presentation

$$
\left.G_{L}=\pi_{1}\left(S^{3} \backslash \hat{\sigma}\right)=\left\langle x_{1}, \ldots, x_{k}\right| x_{i}=x_{i}^{\sigma}, \text { and } i=1, \ldots, k\right\rangle .
$$

The number of components of the link $L=\hat{\sigma}$ is the number of disjoint cycles in the permutation $\bar{\sigma}$. We will be interested in $n$-component links, that is, the closures of braids $\sigma$ with

$$
\bar{\sigma}=\left(i_{1}, \ldots, i_{k_{1}}\right)\left(i_{k_{1}+1}, \ldots, i_{k_{2}}\right) \cdots\left(i_{k_{n-1}+1}, \ldots, i_{k_{n}}\right),
$$

where $1 \leq k_{1}<k_{2}<\cdots<k_{n}=k$. We define multi-indices $I_{1}, I_{2}, \ldots, I_{n}$ by setting $I_{j}=\left\{i_{k_{j-1}+1}, \ldots, i_{k_{j}}\right\}$ for $j=1, \ldots, n$, and we denote $\bar{\sigma}=\left(I_{1}\right) \cdots\left(I_{n}\right)$. If $L=\ell_{1} \cup \cdots \cup \ell_{n}$ is the closure of a braid $\sigma$, we will assume that the cycles in the permutation $\bar{\sigma}=\left(I_{1}\right) \cdots\left(I_{n}\right)$ are written correspondingly, so that the component $\ell_{j}$ of $L$ corresponds to the braid closure of the strands in $I_{j}$.

1C. The special unitary group. Consider the Lie group $\mathrm{SU}(N)$ of unitary $N \times N$ matrices with determinant one. Recall that $\mathrm{SU}(N)$ has real dimension $N^{2}-1$ and has center isomorphic to $\mathbb{Z}_{N}=\left\{\omega^{d} \mid d \in \mathbb{Z}\right\}$, where $\omega=e^{2 \pi i / N}$. Notice that we are viewing $\mathbb{Z}_{N}$ as the subgroup of $U(1)$ consisting of $N$-th roots of unity, and for this reason we view it as a multiplicative group and identify it with the center of $\mathrm{SU}(N)$ via the map defined by sending $\omega^{d} \mapsto \omega^{d} I$. 
Since every matrix in $\mathrm{SU}(N)$ is diagonalizable, conjugacy classes in $\mathrm{SU}(N)$ are completely determined by their eigenvalues when considered with multiplicities. Given $A \in \mathrm{SU}(N)$ we denote its conjugacy class by $C_{A}$. There is a unique conjugacy class $C_{A}$ which is preserved under multiplication by $\omega=e^{2 \pi i / N}$, and this is the conjugacy class of the diagonal matrix $A$ whose eigenvalues are the $N$ distinct $N$-th roots of $(-1)^{N-1}$. Setting $\xi=e^{2 \pi i / 2 N}$, then $A$ is given by the diagonal matrix $\operatorname{diag}\left(1, \omega, \ldots, \omega^{N-1}\right)$ when $N$ is odd and by $\operatorname{diag}\left(\xi, \omega \xi, \ldots, \omega^{N-1} \xi\right)$ when $N$ is even. In either case, since the eigenvalues of $A$ are all distinct, we see that the stabilizer of $A$ is the standard maximal torus $T^{N-1}$ in $\mathrm{SU}(N)$ and that $C_{A} \cong \mathrm{SU}(N) / T^{N-1}$ is the variety of full flags in $\mathbb{C}^{N}$ and has real dimension $N^{2}-N$.

1D. $\mathbf{S U}(N)$ representations. For a discrete group $G$, let $\mathrm{R}(G)=\operatorname{Hom}(G, \mathrm{SU}(N))$ denote the variety of $\mathrm{SU}(N)$ representations of $G$. For convenience, we set $\mathrm{R}_{k}=$ $\mathrm{R}\left(F_{k}\right)=\mathrm{SU}(N) \times \cdots \times \mathrm{SU}(N)$ to be the variety of $\mathrm{SU}(N)$ representations of the free group $F_{k}$. The faithful representation $\varrho: B_{k} \rightarrow \operatorname{Aut}\left(F_{k}\right)$ induces a representation

$$
\tilde{\varrho}: B_{k} \rightarrow \operatorname{Diff}\left(\mathrm{R}_{k}\right)
$$

given by $\tilde{\varrho}(\sigma)(\alpha)=\alpha \circ \sigma$. We will often abuse notation and simply denote $\tilde{\varrho}(\sigma)$ by $\sigma$. Remark 1.2. [Long 1989, Theorem 2.1] implies that $\tilde{\varrho}$ is defined on $\operatorname{Aut}\left(F_{k}\right)$ and is faithful.

Example 1.3. Consider $\sigma_{1}$ and $\sigma_{2} \in B_{3}$. For $X=\left(X_{1}, X_{2}, X_{3}\right) \in \mathrm{R}_{3}$, we have

$$
\sigma_{1}(X)=\left(X_{2}, X_{2}^{-1} X_{1} X_{2}, X_{3}\right) \text { and } \sigma_{2}(X)=\left(X_{1}, X_{3}, X_{3}^{-1} X_{2} X_{3}\right) .
$$

Using this, one can easily compute that

$$
\sigma_{1}^{2}(X)=\left(X_{2}^{-1} X_{1} X_{2}, X_{2}^{-1} X_{1}^{-1} X_{2} X_{1} X_{2}, X_{3}\right)
$$

and further that

$$
\sigma_{1} \sigma_{2}(X)=\left(X_{2}, X_{3}, X_{3}^{-1} X_{2}^{-1} X_{1} X_{2} X_{3}\right)
$$

and

$$
\sigma_{2} \sigma_{1}(X)=\left(X_{3}, X_{3}^{-1} X_{1} X_{3}, X_{3}^{-1} X_{2} X_{3}\right) .
$$

Using the standard presentation (3) of the link group, we notice that $\mathrm{R}\left(G_{L}\right)$ can be identified with $\operatorname{Fix}(\sigma) \subset \mathrm{R}_{k}$,

$$
\mathrm{R}\left(G_{L}\right)=\left\{\left(X_{1}, \ldots, X_{k}\right) \in \mathrm{R}_{k} \mid X_{i}=\sigma(X)_{i}\right\} .
$$

A $k$-tuple $\left(X_{1}, \ldots X_{k}\right) \in \mathrm{R}_{k}$ is called reducible if it can be simultaneously conjugated by an element of $\mathrm{SU}(N)$ such that each $X_{i}$ has the form

$$
X_{i}=\left(\begin{array}{cc}
A_{i} & 0 \\
0 & B_{i}
\end{array}\right)
$$


where $A_{i}$ is a block of size $N_{1}$ and $B_{i}$ is a block of size $N_{2}$, and $N_{1}+N_{2}=N$. A $k$-tuple $\left(X_{1}, \ldots X_{k}\right) \in \mathrm{R}_{k}$ is irreducible if it is not reducible.

1E. The wreath product $\mathbb{Z}_{N} 2 \boldsymbol{B}_{\boldsymbol{k}}$. The wreath product $\mathbb{Z}_{N} 2 B_{k}$ is the semidirect product of $B_{k}$ with $\left(\mathbb{Z}_{N}\right)^{k}$, where $B_{k}$ acts on $\left(\mathbb{Z}_{N}\right)^{k}$ by permutation. In other words, $\mathbb{Z}_{N} 2 B_{k}$ consists of pairs $(\varepsilon, \sigma) \in\left(\mathbb{Z}_{N}\right)^{k} \times B_{k}$, and the group structure is given by

$$
(\varepsilon, \sigma) \cdot\left(\varepsilon^{\prime}, \sigma^{\prime}\right)=\left(\varepsilon \bar{\sigma}\left(\varepsilon^{\prime}\right), \sigma \sigma^{\prime}\right) .
$$

Here, $\bar{\sigma}$ acts on $\varepsilon^{\prime}=\left(\varepsilon_{1}^{\prime}, \ldots, \varepsilon_{k}^{\prime}\right)$ by permutation, i.e., $\bar{\sigma}\left(\varepsilon^{\prime}\right)=\left(\varepsilon_{(1)^{\bar{\sigma}}}^{\prime}, \ldots, \varepsilon_{(k)^{\bar{\sigma}}}^{\prime}\right)$ In particular, it follows that $\sigma(\varepsilon X)=\bar{\sigma}(\varepsilon) \sigma(X)$.

We extend the representation (5) to the representation

$$
\tilde{\varrho}: \mathbb{Z}_{N} 2 B_{k} \rightarrow \operatorname{Diff}\left(\mathrm{R}_{k}\right)
$$

defined by sending the pair $(\varepsilon, \sigma)$ to the diffeomorphism $\varepsilon \sigma: \mathrm{R}_{k} \rightarrow \mathrm{R}_{k}$, where

$$
\varepsilon \sigma(X)=\left(\varepsilon_{1} \sigma(X)_{1}, \ldots, \varepsilon_{k} \sigma(X)_{k}\right) .
$$

Thus $\varepsilon$ twists the coordinates of $\sigma(X)$ by elements of the center $\mathbb{Z}_{N}$.

Example 1.4. For $X=\left(X_{1}, X_{2}, X_{3}\right) \in \mathrm{R}_{3}$ and $\varepsilon=\left(\varepsilon_{1}, \varepsilon_{2}, \varepsilon_{3}\right) \in\left(\mathbb{Z}_{N}\right)^{3}$, we have

$$
\left(\varepsilon \sigma_{1}\right)\left(X_{1}, X_{2}, X_{3}\right)=\left(\varepsilon_{1} X_{2}, \varepsilon_{2} X_{2}^{-1} X_{1} X_{2}, \varepsilon_{3} X_{3}\right)
$$

and

$$
\sigma_{1}(\varepsilon X)=\overline{\sigma_{1}}(\varepsilon) \sigma_{1}(X)=\left(\varepsilon_{2} X_{2}, \varepsilon_{1} X_{2}^{-1} X_{1} X_{2}, \varepsilon_{1} X_{1}, \varepsilon_{3} X_{3}\right)
$$

\section{Projective representations of the link group}

Our goal in this paper is to define invariants of $L$, and we will do so by performing a signed count of certain irreducible projective $\mathrm{SU}(N)$ representations.

2A. Projective representations. Suppose $\sigma \in B_{k}$ is a braid whose closure $\hat{\sigma}$ is a link $L$ in $S^{3}$. For any $k$-tuple $\varepsilon=\left(\varepsilon_{1}, \ldots, \varepsilon_{k}\right) \in\left(\mathbb{Z}_{N}\right)^{k}$, an element $X=$ $\left(X_{1}, \ldots, X_{k}\right) \in \mathrm{SU}(N)^{k}$ in $\operatorname{Fix}(\varepsilon \sigma)$ determines a $\mathrm{PU}(N)$ representation of the link group $G_{L}$, i.e., a homomorphism $\tilde{\alpha}: G_{L} \rightarrow \operatorname{PU}(N)$. To see this, note that for any $X \in \operatorname{Fix}(\varepsilon \sigma)$, since $\varepsilon_{i} \sigma(X)_{i}=X_{i}$ holds in $\operatorname{SU}(N)$ and $\varepsilon_{i} \in \mathbb{Z}_{N}$ is central, the equation $\sigma(\tilde{X})_{i}=\tilde{X}_{i}$ holds for the $k$-tuple $\tilde{X} \in \operatorname{PU}(N)^{k}$, which shows that $\tilde{X}$ determines a representation $\tilde{\alpha}: G_{L} \rightarrow \mathrm{PU}(N)$.

Given a discrete group $G$, we define a projective representation of $G$ to be a function (not a homomorphism!) $\alpha: G \rightarrow \operatorname{SU}(N)$ such that $\alpha(g h) \alpha(h)^{-1} \alpha(g)^{-1} \in \mathbb{Z}_{N}$ for all $g, h \in G$. For any projective representation $\alpha: G \rightarrow \mathrm{SU}(N)$, its composition with the surjection $\mathrm{Ad}: \mathrm{SU}(N) \rightarrow \mathrm{PU}(N)$ gives rise to a representation $\tilde{\alpha}=\operatorname{Ad} \alpha: G \rightarrow \operatorname{PU}(N)$, and thus every projective representation $\alpha: G \rightarrow \operatorname{SU}(N)$ is the lift of an honest representation $\tilde{\alpha}: G \rightarrow \mathrm{PU}(N)$. Alternatively, any representation 
$\tilde{\alpha}: G \rightarrow \mathrm{PU}(N)$ can be lifted to a projective representation $\alpha: G \rightarrow \mathrm{SU}(N)$, though the lift is generally not unique.

Given a projective representation $\alpha: G \rightarrow \mathrm{SU}(N)$, we can associate a map $c: G \times G \rightarrow \mathbb{Z}_{N}$ defined by $c(g, h)=\alpha(g h) \alpha(h)^{-1} \alpha(g)^{-1}$. Notice that the map $c$ satisfies the condition that $c(g h, k) c(g, h)=c(g, h k) c(h, k)$ for all $g, h, k \in G$, and hence $c$ is a 2-cocycle of $G$.

For a fixed 2-cocycle $c: G \times G \rightarrow \mathbb{Z}_{N}$, let $\mathrm{PR}_{c}(G)$ denote the set of projective representations $\alpha: G \rightarrow \mathrm{SU}(N)$ whose associated 2-cocycle is $c$. If $G$ is finitely generated with generating set $\left\{g_{1}, \ldots, g_{k}\right\}$, then any projective representation $\alpha \in \mathrm{PR}_{c}(G)$ is completely determined by the 2-cocycle $c$ and the elements $\alpha\left(g_{1}\right), \ldots, \alpha\left(g_{k}\right) \in \mathrm{SU}(N)$, and in this way one can realize $\mathrm{PR}_{c}(G)$ as a subset of $\mathrm{SU}(N)^{k}$. It is a compact real algebraic variety.

2B. Allowable labels and compatible k-tuples. Given a link $L$ in $S^{3}$ with $n$ components, we can write $L=\ell_{1} \cup \cdots \cup \ell_{n}$. An $n$-tuple $a=\left(a_{1}, \ldots, a_{n}\right) \in \mathbb{Z}^{n}$ of integers is called allowable if the following three conditions are satisfied:

(i) $0 \leq a_{i}<N$ for $i=1, \ldots, n$,

(ii) $d=\operatorname{gcd}\left(a_{1}, \ldots, a_{n}\right)$ is relatively prime to $N$,

(iii) $a_{1}+\cdots+a_{n}$ is a multiple of $N$.

An allowable $n$-tuple $\left(a_{1}, \ldots, a_{n}\right)$ is called an $n$-tuple of labels for $L$, and $a_{j}$ is the label corresponding to the $j$-th component $\ell_{j}$ of $L$.

Suppose now that $L$ is the closure of a braid $\sigma \in B_{k}$, and write the permutation $\bar{\sigma}$ as a product $\left(I_{1}\right) \cdots\left(I_{n}\right)$ of disjoint cycles in such a way that $I_{j}$ corresponds to the $j$-th component $\ell_{j}$ of $L$.

Recall that $\omega=e^{2 \pi i / N}$. A $k$-tuple $\varepsilon=\left(\varepsilon_{1}, \ldots, \varepsilon_{k}\right) \in\left(\mathbb{Z}_{N}\right)^{k}$ for $\sigma$ is said to be compatible with the choice of labels $\left(a_{1}, \ldots, a_{n}\right)$ of $L$ if it satisfies the conditions

$$
\prod_{i \in I_{j}} \varepsilon_{i}=\omega^{a_{j}},
$$

for $j=1, \ldots, n$. This effectively labels each strand of the braid $\sigma$ so that, upon closure of the braid, the $j$-th component $\ell_{j}$ of $L$ is assigned the number $a_{j}$ for its label. Note that with this choice $\varepsilon \sigma$ also preserves condition (2) since, by (8) and condition (iii), we have

$$
\begin{aligned}
(\varepsilon \sigma)(X)_{1} \cdots(\varepsilon \sigma)(X)_{k} & =\left(\varepsilon_{1} \cdots \varepsilon_{k}\right) X_{1} \cdots X_{k} \\
& =\left(\omega^{a_{1}} \cdots \omega^{a_{n}}\right) X_{1} \cdots X_{k}=X_{1} \cdots X_{k} .
\end{aligned}
$$

2C. An obstruction to lifting. For $X \in \operatorname{Fix}(\varepsilon \sigma)$ we will show that the associated representation $\tilde{\alpha}: G_{L} \rightarrow \mathrm{PU}(N)$ does not lift to an $\mathrm{SU}(N)$ representation. Essential for this conclusion is that the $k$-tuple $\varepsilon$ is compatible with $a$, the choice of labels 
for $L$. In particular, we use (8) and condition (ii) to give a nonzero obstruction to lifting $\tilde{\alpha}$ to an $\mathrm{SU}(N)$ representation.

Proposition 2.1. The representation $\tilde{\alpha}: G_{L} \rightarrow \mathrm{PU}(N)$ does not lift to an $\mathrm{SU}(N)$ representation.

Proof. Lift $\tilde{\alpha}$ arbitrarily to a map $\alpha: G_{L} \rightarrow \mathrm{SU}(N)$. Since $\alpha$ is a lift of $\tilde{\alpha}$, for each $i$ we see that $\alpha\left(x_{i}\right)=\eta_{i} X_{i}$ for some $\eta_{i} \in \mathbb{Z}_{N}$. Let $\eta=\left(\eta_{1}, \ldots, \eta_{k}\right) \in\left(\mathbb{Z}_{N}\right)^{k}$ be the corresponding $k$-tuple.

We assume that $\alpha$ is a representation. This implies that $\eta X \in \operatorname{Fix}(\sigma)$. Since $X$ is also a fixed point of $\varepsilon \sigma$,

$$
\eta_{i} X_{i}=\sigma\left(\eta_{i} X_{i}\right)=\eta_{(i)^{\bar{\sigma}}} \sigma(X)_{i}=\left(\varepsilon_{i}\right)^{-1} \eta_{(i)^{\bar{\sigma}}} X_{i} .
$$

By condition (ii), some $a_{j} \neq 0$, and we assume without loss of generality that $a_{1} \neq 0$. Consider the component $\ell_{1}$ associated with the multi-index $I_{1}=\left(i_{1}, \ldots, i_{k_{1}}\right)$; then (8) implies that

$$
\eta_{i_{1}}=\left(\varepsilon_{i_{1}}\right)^{-1} \eta_{i_{2}}=\left(\varepsilon_{i_{1}}\right)^{-1}\left(\varepsilon_{i_{2}}\right)^{-1} \eta_{i_{3}}=\cdots=\left(\varepsilon_{i_{1}}\right)^{-1} \cdots\left(\varepsilon_{i_{k}}\right)^{-1} \eta_{i_{1}}=\omega^{-a_{1}} \eta_{i_{1}},
$$

which is a contradiction since $\omega^{-a_{1}} \neq 1$.

2D. Irreducibility for elements in $\operatorname{Fix}(\varepsilon \sigma)$. We now show that for any allowable $n$-tuple $a=\left(a_{1}, \ldots, a_{n}\right) \in \mathbb{Z}^{n}$ of labels and compatible $k$-tuple $\varepsilon=\left(\varepsilon_{1}, \ldots, \varepsilon_{k}\right) \in$ $\left(\mathbb{Z}_{N}\right)^{k}$, every $X \in \operatorname{Fix}(\varepsilon \sigma)$ is irreducible. The key to the proof is condition (ii) on the labels.

Proposition 2.2. If $X \in \operatorname{Fix}(\varepsilon \sigma)$, then $X$ is irreducible.

Proof. Suppose to the contrary that $X \in \operatorname{Fix}(\varepsilon \sigma)$ is reducible, which means that up to conjugation, we can assume

$$
X_{i}=\left(\begin{array}{cc}
A_{i} & 0 \\
0 & B_{i}
\end{array}\right)
$$

where $A_{i}$ has size $N_{1}$ and $B_{i}$ has size $N_{2}$.

The first step is to consider the component $\ell_{1}$ of $L$. It is obtained by closing the strands of $\sigma$ associated with the cycle $I_{1}=\left(i_{1}, \ldots, i_{k_{1}}\right)$ of $\bar{\sigma}$. By (1), there are words $W_{1}, \ldots, W_{k_{1}}$ in $X_{1}, \ldots, X_{k}$ such that

$$
\begin{aligned}
X_{i_{1}} & =\varepsilon_{i_{1}} W_{1} X_{i_{2}} W_{1}^{-1} \\
& =\left(\varepsilon_{i_{1}} \varepsilon_{i_{2}}\right) W_{1} W_{2} X_{i_{3}} W_{2}^{-1} W_{1}^{-1} \\
& \vdots \\
& =\left(\varepsilon_{i_{1}} \cdots \varepsilon_{i_{1}}\right) W_{1} \cdots W_{k_{1}} X_{i_{1}} W_{k_{1}}^{-1} \cdots W_{1}^{-1} \\
& =\omega^{a_{1}} W X_{i_{1}} W^{-1},
\end{aligned}
$$


where the last step follows by setting $W=W_{1} \cdots W_{k_{1}}$ and applying (8).

Since $W$ is a word in the $X_{i}$, and each $X_{i}$ is block diagonal, it follows that $W$ is also block diagonal so we can write

$$
W=\left(\begin{array}{ll}
P & 0 \\
0 & Q
\end{array}\right)
$$

Applying this to the equation above, we see that the same relationship must hold for the blocks, so

$$
A_{i_{1}}=\omega^{a_{1}} P A_{i_{1}} P^{-1},
$$

and taking the determinant of both sides of (10), we see that

$$
\operatorname{det}\left(A_{i_{1}}\right)=\omega^{a_{1} N_{1}} \operatorname{det}\left(A_{i_{1}}\right) .
$$

Since $\operatorname{det}\left(A_{i_{1}}\right) \neq 0$, this implies $\omega^{a_{1} N_{1}}=1$.

Now repeat the argument for the other components of the link $L$. For the component $\ell_{j}$, which is the one obtained by closing the strands of $\sigma$ associated with the cycle $I_{j}$, (8) implies that $\omega^{a_{j} N_{1}}=1$, and we see this holds for each $j=1, \ldots, n$. However, since $\omega=e^{2 \pi i / N}$ is a primitive $N$-th root of unity, this can only happen if $N$ divides $a_{j} N_{1}$ for each $j=1, \ldots, n$. This contradicts condition (ii) on the labels, and we conclude that each $X \in \operatorname{Fix}(\varepsilon \sigma)$ is in fact irreducible.

Remark 2.3. We would like to thank the referee for the following observation. Suppose $L=\ell_{1} \cup \cdots \cup \ell_{n}$ is a link and let $\Lambda_{i} \cong\left\langle\mu_{i}\right\rangle \times\left\langle\lambda_{i}\right\rangle \cong \mathbb{Z} \times \mathbb{Z}$ denote the $i$-th peripheral subgroup of $G_{L}$, where $\mu_{i}$ and $\lambda_{i}$ denote the meridian and longitude, respectively, of $\ell_{i}$. Given a representation $\tilde{\alpha}: G_{L} \rightarrow \operatorname{PU}(N)$, let $\omega(\tilde{\alpha}) \in H^{2}\left(G_{L}, \mathbb{Z}_{N}\right)$ denote the obstruction cocycle, which is related to the commutator pairing of the restriction $\left.\tilde{\alpha}\right|_{\Lambda_{i}}$ as follows. If $\alpha: G_{L} \rightarrow \mathrm{SU}(N)$ is a set-theoretic lift of $\tilde{\alpha}$, then the commutator pairing of $\Lambda_{i}$ is the map $c_{i}: \Lambda_{i} \times \Lambda_{i} \rightarrow \mathbb{Z}_{N}$, given by $c_{i}(x, y)=[\alpha(x), \alpha(y)]$. Since $\Lambda_{i}$ is free abelian of rank two,

$$
\theta: H^{2}\left(\Lambda_{i}, \mathbb{Z}_{N}\right) \stackrel{\sim}{\longrightarrow} \operatorname{Hom}\left(\Lambda_{i} \wedge \Lambda_{i}, \mathbb{Z}_{N}\right) \cong \mathbb{Z}_{N},
$$

and one can show that $\theta\left(\omega\left(\left.\tilde{\alpha}\right|_{\Lambda_{i}}\right)\right)=c_{i}$.

In the previous proof, the element $W=W_{1} \ldots W_{k_{1}}$ is the image of the longitude $\lambda_{1}$ of $\ell_{1}$, thus our computation that $\left[X_{i_{1}}, W\right]=\omega^{a_{1}}$ determined the commutator pairing $c_{1}=\theta\left(\omega\left(\left.\tilde{\alpha}\right|_{\Lambda_{1}}\right)\right)$ by showing that $c_{1}\left(\mu_{1}, \lambda_{1}\right)=\omega^{a_{1}}$. The labels $a_{1}, \ldots, a_{n}$ thus determine the commutator pairings associated to the peripheral subgroups $\Lambda_{1}, \ldots, \Lambda_{n}$. 


\section{The link invariants}

Throughout this section, we assume that $\sigma$ is a braid with closure $\hat{\sigma}=L$, a link with $n$ components $L=\ell_{1} \cup \cdots \cup \ell_{n}$, and that $a=\left(a_{1}, \ldots, a_{n}\right)$ is an $n$-tuple of allowable labels, with $a_{j}$ the label for the component $\ell_{j}$.

In this section we define $h_{N, a}(\varepsilon \sigma)$ for compatible $k$-tuples $\varepsilon$, and we show that it gives rise to an invariant of $n$-component links in $S^{3}$.

We define $h_{N, a}(\varepsilon \sigma)$ as an algebraic count of certain projective $\mathrm{SU}(N)$ representations in $\operatorname{Fix}(\varepsilon \sigma)$, namely those that satisfy the monodromy condition $X_{i} \in C_{A}$. In other words, we require each $X_{i}$ to be in the conjugacy class of matrices with characteristic polynomial $p_{A}(t)=t^{N}+(-1)^{N}$.

We will first show that $h_{N, a}(\varepsilon \sigma)$ is independent of choice of $\varepsilon$, and then we prove that $h_{N, a}(\varepsilon \sigma)$ gives rise to a well-defined invariant of the underlying link $L$ by showing that it is invariant under the Markov moves.

3A. The definition of $h_{N, a}(\varepsilon \sigma)$. Recall that $A$ is the diagonal matrix consisting of the $N$-th roots of $(-1)^{N-1}$, i.e.,

$$
A= \begin{cases}\operatorname{diag}\left(1, \omega, \ldots, \omega^{N-1}\right) & \text { if } N \text { is odd } \\ \operatorname{diag}\left(\xi, \omega \xi, \ldots, \omega^{N-1} \xi\right) & \text { if } N \text { is even. }\end{cases}
$$

We impose the following monodromy condition and restrict to $k$-tuples lying in the subset $Q_{k} \subset \mathrm{R}_{k}$ given by

$$
Q_{k}=\left\{\left(X_{1}, \ldots, X_{k}\right) \in \mathrm{R}_{k} \mid X_{i} \in C_{A}\right\} .
$$

Since $Q_{k}=\left(C_{A}\right)^{k}$ is a just a $k$-fold product of $C_{A}$, we see that $Q_{k}$ is a manifold of dimension $k\left(N^{2}-N\right)$.

Let $\Delta_{k}=\{(X, X)\} \subset Q_{k} \times Q_{k}$ be the diagonal and $\Gamma_{\varepsilon \sigma}=\{(X, \varepsilon \sigma(X))\} \subset Q_{k} \times Q_{k}$ be the graph of $\varepsilon \sigma$. Notice that we can identify points in the intersection $\Delta_{k} \cap \Gamma_{\varepsilon \sigma}$ with elements in $Q_{k} \cap \operatorname{Fix}(\varepsilon \sigma)$.

For certain choices of labels, it will follow that $\operatorname{Fix}(\varepsilon \sigma) \subset Q_{k}$, i.e., that these monodromy conditions are automatically satisfied. This will occur whenever the labels have the property that each $a_{i}$ is relatively prime to $N$. For a simple example, suppose $N$ is prime, $n$ is a positive multiple of $N$, and $d$ is any positive integer less than $N$. Then one can easily verify that $a=(d, d, \ldots, d)$ is an allowable $n$-tuple of labels, and the next result implies that $\operatorname{Fix}(\varepsilon \sigma) \subset Q_{k}$ for any $k$-tuple $\varepsilon=\left(\varepsilon_{1}, \ldots, \varepsilon_{k}\right)$ compatible with these labels.

Proposition 3.1. Suppose $\left(a_{1}, \ldots, a_{n}\right)$ is an allowable $n$-tuple of labels such that each $a_{j}$ is relatively prime to $N$, and suppose $\varepsilon=\left(\varepsilon_{1}, \ldots, \varepsilon_{k}\right) \in\left(\mathbb{Z}_{N}\right)^{k}$ is compatible with a. Then $\operatorname{Fix}(\varepsilon \sigma) \subset Q_{k}$, i.e., if $X \in \operatorname{Fix}(\varepsilon \sigma)$, then each $X_{j}$ is conjugate to $A$.

Proof. The condition on $a_{j}$ ensures that $\omega^{a_{j}}$ generates $\mathbb{Z}_{N}$ for each $j=1, \ldots, n$, where $\omega=e^{2 \pi i / N}$. Write the induced permutation $\bar{\sigma}=\left(I_{1}\right) \cdots\left(I_{n}\right)$ as a product of 
disjoint cycles, where $I_{j}$ corresponds to the $j$-th component of $L=\hat{\sigma}$. Then for any $i \in I_{j}$, we can apply the same argument used to prove Proposition 2.2 to see that

$$
X_{i}=\omega^{a_{j}} W X_{i} W^{-1} .
$$

Thus, the set of eigenvalues of $X_{i}$ is invariant under multiplication by $\omega^{a_{j}}$, and this shows the eigenvalues of $X$ are given by the set

$$
\left\{\xi, \omega^{a_{j}} \xi, \ldots, \omega^{(N-1) a_{j}} \xi\right\}=\left\{\xi, \omega \xi, \ldots, \omega^{N-1} \xi\right\}
$$

for some $\xi$ satisfying $\xi^{N}=(-1)^{N-1}$. When $N$ is odd, one can take $\xi=1$, and when $N$ is even, one can take $\xi=e^{2 \pi i / 2 N}$. This shows that $X_{i}$ is conjugate to $A$.

Alternative argument: consider the characteristic polynomial of both sides of the above equation; we see that

$$
p_{X_{i}}(t)=p_{\omega^{a_{j}} X_{i}}(t)=p_{X_{i}}\left(\omega^{-a_{j}} t\right) .
$$

Since $\omega^{-a_{j}}$ has order $N, p_{X_{i}}(t)$ must be a polynomial in $t^{N}$, and indeed the only possibility is that $p_{X_{i}}(t)=t^{N}+(-1)^{N}$.

We define

$$
H_{k}=\left\{(X, Y) \in Q_{k} \times Q_{k} \mid X_{1} \cdots X_{k}=Y_{1} \cdots Y_{k}\right\},
$$

and we note that $H_{k}$ is not a manifold because of the presence of reducibles. Recall that $(X, Y) \in Q_{k} \times Q_{k}$ is called reducible if all $X_{i}$ and $Y_{i}$ can be simultaneously conjugated into block diagonal form as in (6). We note that the subset $S_{k} \subset Q_{k} \times Q_{k}$ of reducibles is closed, and that $\left(Q_{k} \times Q_{k}\right)^{*}=\left(Q_{k} \times Q_{k}\right) \backslash S_{k}$ is an open manifold of dimension $2 k\left(N^{2}-N\right)$.

Proposition 3.2. The subset $H_{k}^{*}=H_{k} \backslash S_{k}$ of irreducible representations is an open manifold of dimension $2 k\left(N^{2}-N\right)-\left(N^{2}-1\right)$.

Proof. Clearly $H_{k}^{*}=f^{-1}(I)$, where $f:\left(Q_{k} \times Q_{k}\right)^{*} \rightarrow \mathrm{SU}(N)$ is the map defined by $f(X, Y)=X_{1} \cdots X_{k} Y_{k}^{-1} \cdots Y_{1}^{-1}$. We will show that $I$ is a regular value of $f$, i.e., that $d f_{(X, Y)}$ is surjective for all $(X, Y) \in f^{-1}(I)$. It is enough to prove this statement for the map $f: Q_{\ell}^{*} \rightarrow \mathrm{SU}(N)$ given by $f\left(X_{1}, \ldots, X_{\ell}\right)=X_{1} \cdots X_{\ell}$.

Clearly the matrix $A$, since it is diagonal, lies on the standard maximal torus $T^{N-1} \subset \mathrm{SU}(N)$ with Lie algebra

$$
\mathfrak{t}=\left\{\left(\begin{array}{ccc}
i a_{1} & & 0 \\
& \ddots & \\
0 & & i a_{N}
\end{array}\right) \mid a_{1}+\cdots+a_{N}=0\right\} .
$$

Since $A$ has the standard maximal torus as its stabilizer group, we can identify the tangent space $T_{A}\left(C_{A}\right)$ with the orthogonal complement $\mathfrak{t}^{\perp}$ in $\mathfrak{s u}(N)$, which is the 
subspace

$$
\mathfrak{t}^{\perp}=\left\{\left(\begin{array}{cccc}
0 & z_{12} & \ldots & z_{1, N} \\
-\bar{z}_{12} & 0 & \ddots & \vdots \\
\vdots & \ddots & \ddots & z_{N-1, N} \\
-\bar{z}_{1, N} & \ldots & -\bar{z}_{N-1, N} & 0
\end{array}\right) \mid z_{i j} \in \mathbb{C}\right\} .
$$

There is a similar decomposition of $\mathfrak{s u}(N)$ at each $X_{i}$ in the $\ell$-tuple $\left(X_{1}, \ldots, X_{\ell}\right)$. Because each $X_{i}$ has $N$ distinct eigenvalues, it lies on a unique maximal torus $T_{i} \cong T^{N-1}$ in $\mathrm{SU}(N)$. We let $\mathfrak{t}_{i} \subset \mathfrak{s u}(N)$ denote the corresponding Lie subalgebra, which is the Lie algebra of the stabilizer group of $X_{i}$. Using the decomposition $\mathfrak{s u}(N)=\mathfrak{t}_{i} \oplus \mathfrak{t}_{i}^{\perp}$, we can identify the tangent space $T_{X_{i}}\left(C_{A}\right)$ with $\mathfrak{t}_{i}^{\perp} X_{i}$, the right translation of the subspace $\mathfrak{t}_{i}^{\perp} \subset \mathfrak{s u}(N)$ by $X_{i}$. It is helpful to note that, in terms of the specific subspaces identified above, we have $\mathfrak{t}_{i}=\operatorname{Ad}_{P_{i}} \mathfrak{t}$ and $\mathfrak{t}_{i}^{\perp}=\operatorname{Ad}_{P_{i}} \mathfrak{t}^{\perp}$ for any matrix $P_{i} \in \mathrm{SU}(N)$ such that $X_{i}=P_{i} A P_{i}^{-1}$.

Using the fact that $\mathfrak{t}_{i}$ is the Lie algebra of the stabilizer subgroup of $X_{i}$, one can see that irreducibility of the $\ell$-tuple $\left(X_{1}, \ldots, X_{\ell}\right)$ is equivalent to the condition that $\mathfrak{t}_{1} \cap \cdots \cap \mathfrak{t}_{\ell}=\{0\}$.

For $u_{i} \in \mathfrak{t}_{i}^{\perp}$, we set $x_{i}=u_{i} X_{i} \in \mathfrak{t}_{i}^{\perp} X_{i}=T_{X_{i}}\left(C_{A}\right)$. Differentiating and using the fact that $X_{1} \cdots X_{\ell}=I$, we obtain

$$
\begin{aligned}
\frac{d}{d t}\left(X_{1}+t x_{1}\right)\left(X_{2}+t x_{2}\right) \cdots & \left.\left(X_{\ell}+t x_{\ell}\right)\right|_{t=0} \\
& =x_{1} X_{2} \cdots X_{\ell}+X_{1} x_{2} X_{2} \cdots X_{\ell}+\cdots+X_{1} \cdots X_{\ell-1} x_{\ell} \\
& =u_{1}+X_{2} u_{2} X_{2}^{-1}+\cdots+\left(X_{2} \cdots X_{\ell}\right) u_{\ell}\left(X_{\ell}^{-1} \cdots X_{2}^{-1}\right) .
\end{aligned}
$$

In order to show that the map $d f_{X}$ is onto, we claim that, given any $v \in \mathfrak{s u}(N)$, we can find $u_{i} \in \mathfrak{t}_{i}^{\perp}$ for $i=1, \ldots, \ell$ such that

$$
v=u_{1}+X_{2} u_{2} X_{2}^{-1}+\cdots+\left(X_{2} \cdots X_{\ell}\right) u_{\ell}\left(X_{\ell}^{-1} \cdots X_{2}^{-1}\right) .
$$

Notice that we can solve (11) for any

$$
v \in \mathfrak{t}_{1}^{\perp} \cap\left(X_{1} \mathfrak{t}_{2}^{\perp} X_{1}^{-1}\right) \cap \cdots \cap\left(X_{1} \cdots X_{\ell-1}\right) \mathfrak{t}_{\ell}^{\perp}\left(X_{\ell-1}^{-1} \cdots X_{1}^{-1}\right) .
$$

Notice further that since $\mathfrak{t}_{i}$ is the Lie algebra of the maximal torus containing $X_{i}$, we have $\mathfrak{t}_{i} \cap \mathfrak{t}_{i+1}=t_{i} \cap\left(X_{i} \mathfrak{t}_{i+1} X_{i}^{-1}\right)$. More generally, for any subspace $V \subset \mathfrak{s u}(N)$, we have $\mathfrak{t}_{i} \cap V=t_{i} \cap\left(X_{i} V X_{i}^{-1}\right)$. Repeated application gives that

$$
\begin{aligned}
\mathfrak{t}_{1} \cap \cdots \cap \mathfrak{t}_{\ell} & =\mathfrak{t}_{1} \cap \cdots \cap\left(X_{\ell-1} \mathfrak{t}_{\ell} X_{\ell-1}^{-1}\right) \\
& =\mathfrak{t}_{1} \cap \cdots \cap\left(X_{\ell-2} \mathfrak{t}_{\ell-1} X_{\ell-2}^{-1}\right) \cap\left(X_{\ell-2} X_{\ell-1} \mathfrak{t}_{\ell} X_{\ell-1}^{-1} X_{\ell-2}^{-1}\right) \\
& \vdots \\
& =\mathfrak{t}_{1} \cap\left(X_{1} \mathfrak{t}_{2} X_{1}^{-1}\right) \cap \cdots \cap\left(X_{1} \cdots X_{\ell-1}\right) \mathfrak{t}_{\ell}\left(X_{\ell-1}^{-1} \cdots X_{1}^{-1}\right) .
\end{aligned}
$$


The condition of irreducibility implies that $\mathfrak{t}_{1} \cap \cdots \cap \mathfrak{t}_{\ell}=\{0\}$, and it then follows from the above that (11) can be solved for any $v \in \mathfrak{s u}(N)$. This concludes the argument that $d f_{X}$ is a surjection whenever the $\ell$-tuple $X=\left(X_{1}, \ldots, X_{\ell}\right)$ is irreducible.

Since both $\Delta_{k}$ and $\Gamma_{\varepsilon \sigma}$ preserve the product $X_{1} \cdots X_{k}$ (see (9)), we can restrict from $Q_{k} \times Q_{k}$ to $H_{k}$.

Now we are in a position to define the invariant $h_{N, a}(\varepsilon \sigma)$. Set $\Gamma_{\varepsilon \sigma}^{*}=\Gamma_{\varepsilon \sigma} \cap H_{k}^{*}$ and $\Delta_{k}^{*}=\Delta_{k} \cap H_{k}^{*}$. Since $S_{k}$ is closed, it follows that both $\Gamma^{*}$ and $\Delta_{k}^{*}$ are open submanifolds of $H_{k}^{*}$ of dimension $k\left(N^{2}-N\right)$.

Both $\Delta_{k}$ and $\Gamma_{\varepsilon \sigma}$ are compact, and so is their intersection $\Delta_{k} \cap \Gamma_{\varepsilon \sigma}$. Consequently, as Proposition 2.2 implies that every point in this intersection is irreducible, we have the following result.

Corollary 3.3. The intersection $\Delta_{k}^{*} \cap \Gamma_{\varepsilon \sigma}^{*} \subset H_{k}^{*}$ is compact.

The group $\mathrm{PU}(N)$ acts freely by conjugation on each of $H_{k}^{*}, \Delta_{k}^{*}$, and $\Gamma_{\varepsilon \sigma}^{*}$, and the quotients by this action are the manifolds we denote as

$$
\hat{H}_{k}=H_{k}^{*} / \mathrm{PU}(N), \quad \hat{\Delta}_{k}=\Delta_{k}^{*} / \mathrm{PU}(N), \quad \text { and } \quad \hat{\Gamma}_{\varepsilon \sigma}=\Gamma_{\varepsilon \sigma}^{*} / \mathrm{PU}(N) .
$$

Here the dimension of $\hat{H}_{k}$ equals $2 k\left(N^{2}-N\right)-2\left(N^{2}-1\right)$, and both $\hat{\Delta}_{k}$ and $\hat{\Gamma}_{\varepsilon \sigma}$ are half-dimensional submanifolds of $\hat{H}_{k}$. Since the intersection $\hat{\Delta}_{k} \cap \hat{\Gamma}_{\varepsilon \sigma}$ is compact, we can deform $\hat{\Gamma}_{\varepsilon \sigma}$ into a submanifold $\tilde{\Gamma}_{\varepsilon \sigma}$ using an isotopy with compact support so that the intersection $\hat{\Delta}_{k} \cap \tilde{\Gamma}_{\varepsilon \sigma}$ is transverse and consists of finitely many points. Define

$$
h_{N, a}(\varepsilon \sigma)=\#_{\hat{H}_{k}}\left(\hat{\Delta}_{k} \cap \tilde{\Gamma}_{\varepsilon \sigma}\right)
$$

as the oriented intersection number. We will describe the orientations in the following subsection. The intersection number $h_{N, a}(\varepsilon \sigma)$ is independent of the choice of isotopy of $\hat{\Gamma}_{\varepsilon \sigma}$, and we denote

$$
h_{N, a}(\varepsilon \sigma)=\left\langle\hat{\Delta}_{k}, \hat{\Gamma}_{\varepsilon \sigma}\right\rangle_{\hat{H}_{k}}
$$

3B. Orientations. The following argument is similar to the one found in [Harper and Saveliev 2010, Section 3.4], and we include it here for completeness.

First, observe that the conjugacy class $C_{A} \subset \mathrm{SU}(N)$ is orientable, which follows for instance by identifying it with a flag variety. So choose an orientation for $C_{A}$ and give $Q_{k}=\left(C_{A}\right)^{k}$ and $Q_{k} \times Q_{k}$ the induced product orientations. The diagonal $\Delta_{k}$ and the graph $\Gamma_{\varepsilon \sigma}$ are naturally diffeomorphic to $Q_{k}$ via projection and so an orientation for $Q_{k}$ determines orientations for both $\Delta_{k}$ and $\Gamma_{\varepsilon \sigma}$.

Using the standard orientation of $\mathrm{SU}(N)$, we obtain an orientation on $H_{k}^{*}=$ $f^{-1}(I)$ using the base-fiber rule. Since the adjoint action of $\mathrm{PU}(N)$ on $C_{A}$ is orientation preserving, the quotients $\hat{H}_{k}, \hat{\Delta}_{k}$, and $\hat{\Gamma}_{\varepsilon \sigma}$ are all orientable, and we orient them using the base-fiber rule. 
Reversing the orientation of $C_{A}$ reverses the orientation of $Q_{k}$ only when $k$ is odd, and in this case it reverses the orientations of both $\hat{\Delta}_{k}$, and $\hat{\Gamma}_{\varepsilon \sigma}$ but it does not affect the oriented intersection number $\left\langle\hat{\Delta}_{k}, \hat{\Gamma}_{\varepsilon \sigma}\right\rangle_{\hat{H}_{k}}$. This shows that the intersection number is actually independent of the choice of orientation on the conjugacy class $C_{A}$.

3C. Independence of $\boldsymbol{\varepsilon}$. The next result shows that $h_{N, a}(\varepsilon \sigma)$ is independent of the choice of $\varepsilon$ compatible with $a$.

Proposition 3.4. Fix a link $L$ with $n>1$ components and an allowable $n$-tuple $a=$ $\left(a_{1}, \ldots, a_{n}\right)$ of labels. Fix also a braid $\sigma \in B_{k}$ with closure $\hat{\sigma}=L$. If $\varepsilon, \varepsilon^{\prime} \in\left(\mathbb{Z}_{N}\right)^{k}$ are $k$-tuples compatible with a, i.e., satisfying (8), then $h_{N, a}(\varepsilon \sigma)=h_{N, a}\left(\varepsilon^{\prime} \sigma\right)$.

Proof. We will define an orientation preserving automorphism $\varphi: \hat{H}_{k} \rightarrow \hat{H}_{k}$ such that $\varphi\left(\hat{\Delta}_{k}\right)=\hat{\Delta}_{k}$ and $\varphi\left(\hat{\Gamma}_{\varepsilon \sigma}\right)=\hat{\Gamma}_{\varepsilon^{\prime} \sigma}$. Write the permutation

$$
\bar{\sigma}=\left(i_{1}, \ldots, i_{k_{1}}\right)\left(i_{k_{1}+1}, \ldots, i_{k_{2}}\right) \cdots\left(i_{k_{n-1}+1}, \ldots, i_{k_{n}}\right)
$$

as a product of disjoint cycles as in (4) and define $\delta=\left(\delta_{1}, \ldots, \delta_{k}\right) \in\left(\mathbb{Z}_{N}\right)^{k}$ recursively with initial values

$$
\delta_{i_{1}}=1=\delta_{i_{k_{1}+1}}=\cdots=\delta_{i_{k_{n-1}+1}}
$$

and by setting

$$
\delta_{(j)^{\bar{\sigma}}}=\delta_{j} \varepsilon_{j}\left(\varepsilon_{j}^{\prime}\right)^{-1} .
$$

Writing $\bar{\sigma}$ as a product of disjoint cycles as in (12) and noting that $\varepsilon$ and $\varepsilon^{\prime}$ both satisfy (8), repeated application of the recursion (14) shows that the definition of $\delta=\left(\delta_{1}, \ldots, \delta_{k}\right)$ is compatible with the initial values taken in (13).

Define the diffeomorphism $\tau: Q_{k} \rightarrow Q_{k}$ by

$$
\tau(X)=\delta X=\left(\delta_{1} X_{1}, \ldots, \delta_{k} X_{k}\right) .
$$

Note that $\tau$ may be orientation preserving or reversing. Furthermore, $\tau$ preserves irreducibility and commutes with conjugation.

Consider the product map $\tau \times \tau: Q_{k} \times Q_{k} \rightarrow Q_{k} \times Q_{k}$. Observe that $\tau \times \tau$ preserves the orientation of $Q_{k} \times Q_{k}$ and hence the induced map $\varphi: \hat{H}_{k} \rightarrow \hat{H}_{k}$ is orientation preserving.

Since $\tau$ may be orientation reversing, $\varphi$ restricted to $\hat{\Delta}_{k}$ or $\hat{\Gamma}_{\varepsilon \sigma}$ may be orientation reversing. The key observation is that if $\varphi$ is orientation reversing on one, then it must be orientation reversing on the other. Hence, $\varphi$ preserves the intersection number $h_{N, a}(\varepsilon \sigma)$,

$$
\left\langle\hat{\Delta}_{k}, \hat{\Gamma}_{\varepsilon \sigma}\right\rangle_{\hat{H}_{k}}=\left\langle\varphi\left(\hat{\Delta}_{k}\right), \varphi\left(\hat{\Gamma}_{\varepsilon \sigma}\right)\right\rangle_{\hat{H}_{k}}
$$


Clearly, $\varphi\left(\hat{\Delta}_{k}\right)=\hat{\Delta}_{k}$, so to finish off the proof we check that $\varphi\left(\hat{\Gamma}_{\varepsilon \sigma}\right)=\hat{\Gamma}_{\varepsilon^{\prime} \sigma}$, or that the pair $(\delta X, \delta \varepsilon \sigma(X)) \in \hat{\Gamma}_{\varepsilon^{\prime} \sigma}$. By the calculation

$$
(\delta X, \delta \varepsilon \sigma(X))=\left(\delta X, \delta \varepsilon \sigma\left(\delta^{N-1} \delta X\right)\right)=\left(\delta X, \delta \varepsilon \bar{\sigma}\left(\delta^{N-1}\right) \sigma(\delta X)\right),
$$

this will follow once we verify that $\delta \varepsilon \bar{\sigma}\left(\delta^{N-1}\right)=\varepsilon^{\prime}$. Since $\delta_{j}^{N-1}=\delta_{j}^{-1}$, this is equivalent to showing that $\delta_{j} \varepsilon_{j}=\bar{\sigma}\left(\delta_{j}\right) \varepsilon_{j}^{\prime}$ for $j=1, \ldots, k$, which follows directly from (14), and this completes the proof of the proposition.

3D. Independence under Markov moves. Based on the previous result, we denote $h_{N, a}(\varepsilon \sigma)$ by $h_{N, a}(\sigma)$ assuming that a choice of compatible $\varepsilon$ has been made. In this subsection, we show that $h_{N, a}$ defines an invariant of $n$-component links, and this is achieved by showing that $h_{N, a}(\sigma)$ is invariant under the Markov moves.

Recall that two braids $\sigma, \tau \in B_{k}$ have isotopic closures $\hat{\sigma}=\hat{\tau}$ if and only if $\sigma$ can be obtained from $\tau$ by a finite sequence of Markov moves; see for example [Birman 1974]. The first Markov move replaces $\sigma \in B_{k}$ by $\xi^{-1} \sigma \xi \in B_{k}$ for $\xi \in B_{k}$, and the second Markov move exchanges $\sigma \in B_{k}$ with $\sigma \sigma_{k}^{ \pm 1} \in B_{k+1}$.

The following propositions give the $\mathrm{SU}(N)$ analogues of the $\mathrm{SU}(2)$ results in [Harper and Saveliev 2010, Propositions 4.2 and 4.3]; see the proof of [Lin 1992, Theorem 1.8].

Proposition 3.5. The quantity $h_{N, a}(\sigma)$ is invariant under type 1 Markov moves. Proof. Suppose $\sigma \in B_{k}$ is a braid with

$$
\bar{\sigma}=\left(I_{1}\right) \cdots\left(I_{n}\right)=\left(i_{1}, \ldots, i_{k_{1}}\right) \cdots\left(i_{k_{n-1}+1}, \ldots, i_{k_{n}}\right)
$$

in multi-index notation. Given a braid $\xi \in B_{k}$, let $\sigma^{\prime}=\xi^{-1} \sigma \xi$ and note that $\overline{\sigma^{\prime}}$ has the same cycle structure as $\bar{\sigma}$, in fact it is given by

$$
\overline{\sigma^{\prime}}=\left(I_{1}^{\xi}\right) \cdots\left(I_{n}^{\xi}\right)=\left(\left(i_{1}\right)^{\bar{\xi}}, \ldots,\left(i_{k_{1}}\right)^{\bar{\xi}}\right) \cdots\left(\left(i_{k_{n-1}+1}\right)^{\bar{\xi}}, \ldots,\left(i_{k_{n}}\right)^{\bar{\xi}}\right) .
$$

We choose $\varepsilon^{\prime} \in\left(\mathbb{Z}_{N}\right)^{k}$ compatible with the given labels, which means that $\varepsilon^{\prime}$ satisfies (8) with respect to the braid $\sigma^{\prime}$, namely

$$
\prod_{i \in I_{j}^{\xi}} \varepsilon_{i}^{\prime}=\omega^{a_{j}}
$$

holds for $j=1, \ldots, k$. Notice that if we define the $k$-tuple $\varepsilon$ by setting $\varepsilon_{i}=\varepsilon_{(i)^{\bar{\xi}}}^{\prime}$, then one can show that $\varepsilon$ satisfies (8) with respect to $\bar{\sigma}=\left(I_{1}\right) \cdots\left(I_{n}\right)$; hence $\varepsilon$ is also compatible with the given labels.

The braid $\xi$ determines a map $\xi: Q_{k} \rightarrow Q_{k}$, and since it acts by permutation and conjugation on each of the factors in $Q_{k}=C_{A} \times \cdots \times C_{A}$, the fact that $C_{A}$ is even-dimensional implies that this map is orientation preserving. This induces the map $\xi \times \xi$ on $Q_{k} \times Q_{k}$ preserving irreducibility, commuting with the adjoint action 
of $\mathrm{PU}(n)$, and preserving (2), thus we obtain a well-defined orientation preserving $\operatorname{map} \xi \times \xi: \hat{H}_{k} \rightarrow \hat{H}_{k}$.

Clearly, $(\xi \times \xi)\left(\hat{\Delta}_{k}\right)=\hat{\Delta}_{k}$, so the diagonal is preserved, and we consider the effect of $\xi \times \xi$ on the graph $\hat{\Gamma}_{\varepsilon^{\prime} \sigma^{\prime}}$. If $\left(X, \varepsilon^{\prime} \sigma^{\prime}(X)\right) \in \hat{\Gamma}_{\varepsilon^{\prime} \sigma^{\prime}}$, then

$$
(\xi \times \xi)\left(X, \varepsilon^{\prime} \sigma^{\prime}(X)\right)=(\xi \times \xi)\left(X, \varepsilon^{\prime} \xi^{-1} \sigma \xi(X)\right)=\left(\xi(X), \xi\left(\varepsilon^{\prime}\right) \sigma \xi(X)\right) \in \hat{\Gamma}_{\varepsilon \sigma},
$$

since $\left.\xi\left(\varepsilon^{\prime}\right)_{i}=\varepsilon_{(i)}^{\prime}\right)^{\bar{\xi}}=\varepsilon_{i}$. Thus $(\xi \times \xi)\left(\hat{\Gamma}_{\varepsilon^{\prime} \sigma^{\prime}}\right)=\hat{\Gamma}_{\varepsilon \sigma}$, and we see that

$$
\begin{aligned}
h_{N, a}\left(\sigma^{\prime}\right)=\left\langle\hat{\Delta}_{k}, \hat{\Gamma}_{\varepsilon^{\prime} \sigma^{\prime}}\right\rangle_{\hat{H}_{k}} & =\left\langle(\xi \times \xi)\left(\hat{\Delta}_{k}\right),(\xi \times \xi)\left(\hat{\Gamma}_{\varepsilon^{\prime} \sigma^{\prime}}\right)\right\rangle_{\xi \times \xi\left(\hat{H}_{k}\right)} \\
& =\left\langle\hat{\Delta}_{k}, \hat{\Gamma}_{\varepsilon \sigma}\right\rangle_{\hat{H}_{k}}=h_{N, a}(\sigma) .
\end{aligned}
$$

The next result is established using the same argument that is used to prove [Harper and Saveliev 2010, Proposition 4.3; Lin 1992, Theorem 1.8. We leave the details of the proof to the reader.

Proposition 3.6. The quantity $h_{N, a}(\sigma)$ is invariant under type 2 Markov moves.

\section{Computations}

In this section, we perform computations of $h_{N, a}(L)$ for various links $L$ and we prove a vanishing condition for $h_{N, a}(L)$ for split links.

4A. The Hopf link and chain links. The chain link $L$ is obtained as the closure of the braid $\sigma=\sigma_{1}^{2} \sigma_{2}^{2} \cdots \sigma_{n-1}^{2} \in B_{n}$. In this subsection, we compute $h_{N, a}(L)$ for $L$ the Hopf link and the chain link with $N=n$ components. In particular, if $d$ is chosen relatively prime to $N$ and $a=(d, \ldots, d)$, then we will show that $h_{N, a}(L)=0$ for the chain link with $n>2$ components. For $n=2, L$ is just the Hopf link, which we denote by $H \subset S^{3}$. Harper and Saveliev [2010] proved that $h_{2, a}(H)= \pm 1$ for $a=(1,1)$. We generalize this by showing that $h_{N, a}(H)= \pm 1$ if $a=(d, N-d)$, where $d$ satisfies $1 \leq d<N$ and is relatively prime to $N$.

The next result will be used repeatedly in the computations that follow.

Theorem 4.1. Suppose $N \geq 2$ and set $\omega=e^{2 \pi i / N}$ and $\xi=e^{2 \pi i / 2 N}$. Any pair of matrices $(X, Y) \in \mathrm{SU}(N) \times \mathrm{SU}(N)$ satisfying $[X, Y]=\omega I$ is, up to conjugation, given by

$$
X= \begin{cases}\operatorname{diag}\left(1, \omega, \ldots, \omega^{N-1}\right) & \text { if } N \text { is odd } \\ \operatorname{diag}\left(\xi, \xi \omega, \ldots, \xi \omega^{N-1}\right) & \text { if } N \text { is even }\end{cases}
$$

and

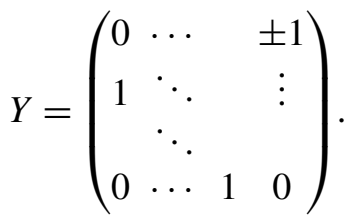


The pair $(X, Y) \in \mathrm{SU}(N) \times \mathrm{SU}(N)$ determines an irreducible projective $\mathrm{SU}(N)$ representation of the free abelian group $\mathbb{Z} \oplus \mathbb{Z}$ of rank 2 .

Proof. First notice that $X Y X^{-1} Y^{-1}=\omega I$ if and only if $Y^{-1} X Y=\omega X$. Every element of $\mathrm{SU}(N)$ is conjugate to a diagonal matrix, and so we can write

$$
X=\left(\begin{array}{ccc}
\lambda_{1} & & 0 \\
& \ddots & \\
0 & & \lambda_{N}
\end{array}\right),
$$

where $\lambda_{i} \in U(1)$ and $\lambda_{1} \cdots \lambda_{N}=1$. However, because $X$ is conjugate to $\omega X$, we must have

$$
\left\{\lambda_{1}, \ldots, \lambda_{N}\right\}=\left\{\omega \lambda_{1}, \ldots, \omega \lambda_{N}\right\} .
$$

Reordering the terms, we can arrange that $\lambda_{i}=\omega^{i-1} \lambda_{1}$ for $i=1, \ldots, N$. Since $\operatorname{det} X=1$, we have $\lambda_{1}^{N}=(-1)^{N-1}$, and so without loss of generality we can take

$$
\lambda_{1}= \begin{cases}1 & \text { if } N \text { is odd } \\ \xi & \text { if } N \text { is even }\end{cases}
$$

This shows that $X$ is of the required form.

Next, observe that $X Y X^{-1} Y^{-1}=\omega I$ if and only if $X Y=\omega Y X$. Writing $Y=\left(y_{i j}\right)$ and comparing the $(i j)$ entries on right and left, it follows that

$$
\omega^{i-1} y_{i j}=y_{i j} \omega^{j} \text {. }
$$

This implies that $y_{i j}=0$ unless $i \equiv j+1 \bmod N$. Furthermore, since $Y$ has only one nonzero entry in each row and column, each entry must lie in $U(1)$ and we find that

$$
Y=\left(\begin{array}{cccc}
0 & \cdots & & \mu_{1} \\
\mu_{2} & \ddots & & \vdots \\
& \ddots & & \\
0 & & \mu_{N} & 0
\end{array}\right)
$$

where $\mu_{i} \in U(1)$ satisfy $\mu_{1} \cdots \mu_{N}=(-1)^{N-1}$ (since $\operatorname{det} Y=1$ ). Because $X$ is diagonal with $N$ distinct eigenvalues, the stabilizer subgroup $\operatorname{Stab}(X)$ is a copy of the standard maximal torus, i.e.,

$$
\operatorname{Stab}(X)=\left\{\operatorname{diag}\left(\theta_{1}, \ldots, \theta_{N}\right) \mid \theta_{i} \in U(1), \theta_{1} \cdots \theta_{N}=1\right\} \cong T^{N-1} .
$$

A matrix $P=\operatorname{diag}\left(\theta_{1}, \ldots, \theta_{N}\right) \in \operatorname{Stab}(X)$ acts on $Y$ by

$$
P Y P^{-1}=\left(\begin{array}{cccc}
0 & \cdots & & \theta_{1} \theta_{N}^{-1} \mu_{1} \\
\theta_{2} \theta_{1}^{-1} \mu_{2} & \ddots & & \vdots \\
& \ddots & & \\
0 & & \theta_{N} \theta_{N-1}^{-1} \mu_{N} & 0
\end{array}\right) .
$$


Setting

$$
\begin{aligned}
\theta_{1} & =\mu_{1}^{-1} \\
\theta_{2} & =\theta_{1} \mu_{2}^{-1}=\mu_{1}^{-1} \mu_{2}^{-1} \\
\theta_{3} & =\theta_{2} \mu_{3}^{-1}=\mu_{1}^{-1} \mu_{2}^{-1} \mu_{3}^{-1} \\
& \vdots \\
\theta_{N} & =\theta_{N-1} \mu_{N}^{-1}=\mu_{1}^{-1} \cdots \mu_{N}^{-1}=(-1)^{N-1},
\end{aligned}
$$

it follows that

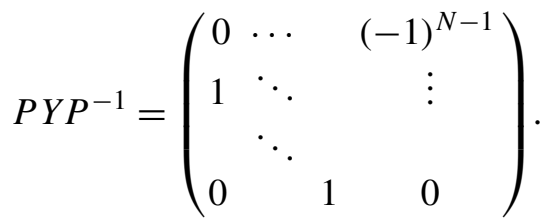

Since $P X P^{-1}=X$, this shows that, up to conjugation, $Y$ is of the required form. Irreducibility of the pair $(X, Y)$ follows from the fact that $\operatorname{Stab}(X) \cap \operatorname{Stab}(Y)=\mathbb{Z}_{N}$.

Remark 4.2. If $X Y X^{-1}=\omega Y$, then $X^{d} Y X^{-d}=\omega^{d} Y$ by induction. This shows that if $(X, Y)$ are as in Theorem 4.1, then $\left(X^{d}, Y\right)$ satisfies $\left[X^{d}, Y\right]=\omega^{d} I$. Using this observation, one can show that solutions $\left(X^{\prime}, Y^{\prime}\right)$ to $\left[X^{\prime}, Y^{\prime}\right]=\omega^{d} I$ are irreducible and unique up to conjugation provided $d$ is relatively prime to $N$. This fails if $d$ is not relatively prime to $N$; when $N=4$ and $d=2$, one can construct nonconjugate families of pairs $(X, Y) \in \mathrm{SU}(4) \times \mathrm{SU}(4)$ satisfying $[X, Y]=-I$. All of these pairs are reducible.

We now use this to evaluate $h_{N, a}(H)$ for the Hopf link $H$.

Proposition 4.3. Suppose $H$ is the Hopf link and $1 \leq d<N$ is relatively prime to $N$. Then $h_{N, a}(H)= \pm 1$ for $a=(d, N-d)$.

Proof. We motivate the proof with the following argument. The Hopf link $H$ has link group $G_{H}=\langle x, y \mid[x, y]=1\rangle$, and Theorem 4.1 implies there is a unique irreducible projective representation $\varrho: G_{H} \rightarrow \mathrm{SU}(N)$ with $[\varrho(x), \varrho(y)]=\omega^{d} I$. Uniqueness of $\varrho$ up to conjugacy implies that $h_{N, a}(H)= \pm 1$.

More precisely, notice that the Hopf link is the closure of the braid $\sigma_{1}^{2} \in B_{2}$ and fix the labels $a=(d, N-d)$ for $H$, where $1 \leq d<N$ is relatively prime to $N$. The braid $\sigma=\sigma_{1}^{2}$ acts on pairs $\left(X_{1}, X_{2}\right) \in \mathrm{R}_{2}=\mathrm{SU}(N) \times \mathrm{SU}(N)$ in the usual way (see Example 1.1), and for $\varepsilon=\left(\varepsilon_{1}, \varepsilon_{2}\right)$ we have

$$
\varepsilon \sigma\left(X_{1}, X_{2}\right)=\left(\varepsilon_{1} X_{2}^{-1} X_{1} X_{2}, \varepsilon_{2} X_{2}^{-1} X_{1}^{-1} X_{2} X_{1} X_{2}\right) .
$$

For $\left(\varepsilon_{1}, \varepsilon_{2}\right)=\left(\omega^{d}, \omega^{N-d}\right)$, one can easily see that $\left(X_{1}, X_{2}\right) \in \operatorname{Fix}(\varepsilon \sigma)$ if and only if $\left[X_{1}, X_{2}\right]=\omega^{d}$. By Theorem 4.1 and the preceding remarks, this equation has 
one solution which is irreducible and unique up to conjugation. Lemma 4.4 below shows that the solution is nondegenerate, and this implies that $h_{N, a}(H)= \pm 1$ for the Hopf link.

The next result establishes the nondegeneracy result required for the above computation of $h_{N, a}(H)$.

Lemma 4.4. Let $H$ be the Hopf link, $G_{H}$ its link group, and $1 \leq d<N$ relatively prime to $N$. Suppose $\varrho: G_{H} \rightarrow \mathrm{SU}(N)$ is the projective representation, unique up to conjugation, of the link group $G_{H}$ with $a=(d, N-d)$. Then $H^{1}\left(G_{H} ; \mathfrak{s u}(N)_{\operatorname{Ad} \varrho}\right)=0$.

Proof. Let $Z=S^{3} \backslash \tau H$ be the link exterior, and recall that the exterior of every nonsplit link in $S^{3}$ is a $K(\pi, 1)$. Thus $H^{i}\left(Z ; \mathfrak{s u}(N)_{\operatorname{Ad} \varrho}\right)=H^{i}\left(G_{H} ; \mathfrak{s u}(N)_{\operatorname{Ad} \varrho}\right)$, where $G_{H}=\pi_{1}(Z)$ is the link group.

For the Hopf link, the link group $G_{H}=\mathbb{Z} \times \mathbb{Z}$ is the free abelian group of rank two. Since $\varrho$ is irreducible, it follows that $H^{0}\left(G_{H} ; \mathfrak{s u}(N)_{\operatorname{Ad} \varrho}\right)=0$, and Poincaré duality implies that $H^{2}\left(G_{H} ; \mathfrak{s u}(N)_{\operatorname{Ad} \varrho}\right)=0$. Using $\chi(Z)=0$, this shows that $H^{1}\left(G_{H} ; \mathfrak{s u}(N)_{\mathrm{Ad} \varrho}\right)=0$, which completes the proof of the lemma.

Next, we consider a chain link $L$ and we establish the following vanishing result for $h_{N, a}(L)$.

Proposition 4.5. Suppose $L$ is a chain link with $n>2$ components and that $n=N$. Then $h_{N, a}(L)=0$ for $a=(d, \ldots, d)$, where $d$ is relatively prime to $N$.

Proof. We start with the chain link $L$ with $n=3$ components. It has link group with presentation

$$
G_{L}=\langle x, y, z \mid[x, y]=1=[y, z]\rangle .
$$

We will parametrize all triples $(X, Y, Z) \in \mathrm{SU}(3) \times \mathrm{SU}(3) \times \mathrm{SU}(3)$ satisfying $[X, Y]=\omega I=[Y, Z]$, and we will use this to show that $h_{3, a}(L)=0$ for $a=(1,1,1)$.

Applying Theorem 4.1, up to conjugacy, there is a unique irreducible pair $(X, Y) \in \mathrm{SU}(3) \times \mathrm{SU}(3)$ satisfying the equation $[X, Y]=\omega I$. This pair is given by

$$
X=\left(\begin{array}{ccc}
1 & 0 & 0 \\
0 & \omega & 0 \\
0 & 0 & \omega^{2}
\end{array}\right) \quad \text { and } \quad Y=\left(\begin{array}{lll}
0 & 0 & 1 \\
1 & 0 & 0 \\
0 & 1 & 0
\end{array}\right)
$$

In a general group, the commutator satisfies the relations

$$
[x, y]^{-1}=[y, x]=y\left[x, y^{-1}\right] y^{-1}=x\left[x^{-1}, y\right] x^{-1} .
$$

Setting $Z=X^{-1}$, this shows that $[Y, Z]=\omega I$, and thus the triple $(X, Y, Z)$ gives rise to a projective representation $\varrho: G_{L} \rightarrow \mathrm{SU}(3)$ satisfying

$$
[\varrho(x), \varrho(y)]=\omega I=[\varrho(y), \varrho(z)] .
$$


If $P \in \operatorname{Stab}(Y)$, then

$$
\left[Y, P Z P^{-1}\right]=P[Y, Z] P^{-1}=\omega I,
$$

so the action of $\operatorname{Stab}(Y)$ on triples, given by $(X, Y, Z) \mapsto\left(X, Y, P Z P^{-1}\right)$, preserves the relations and is nontrivial on conjugacy classes. It follows that the solution set is 2-dimensional and parametrized by $\operatorname{Stab}(Y) / \mathbb{Z}_{3}$, which has Euler characteristic zero since $\operatorname{Stab}(Y) \cong T^{2}$ is a copy of a maximal torus. A calculation similar to the one in the proof of Lemma 4.4 shows that the solution set is a nondegenerate critical submanifold, and a standard argument then shows that its contribution to the invariant is given by plus or minus its Euler characteristic; see the proof of Proposition 8 in [Boden and Herald 1999]. It follows that $h_{3, a}(L)=0$ for $a=(1,1,1)$, and a similar argument shows that $h_{3, a}(L)=0$ for $a=(2,2,2)$.

One can also prove this via a direct approach making use of the fact that $L$ is the closure of the braid $\sigma=\sigma_{1}^{2} \sigma_{2}^{2}$ and parametrizing the fixed point set $\operatorname{Fix}(\varepsilon \sigma)$ as was done for the Hopf link. We leave the details to the reader.

Next, consider the chain link $L$ with 4 components. It has link group with presentation

$$
G_{L}=\langle x, y, z, w \mid[x, y]=1=[y, z]=[z, w]\rangle .
$$

By Theorem 4.1, up to conjugacy, there is a unique irreducible pair $(X, Y) \in$ $\mathrm{SU}(4) \times \mathrm{SU}(4)$ satisfying the equation $[X, Y]=\omega I$. This pair is given by

$$
X=\left(\begin{array}{cccc}
\xi & 0 & 0 & 0 \\
0 & \xi^{3} & 0 & 0 \\
0 & 0 & \xi^{5} & 0 \\
0 & 0 & 0 & \xi^{7}
\end{array}\right) \quad \text { and } \quad Y=\left(\begin{array}{cccc}
0 & 0 & 0 & -1 \\
1 & 0 & 0 & 0 \\
0 & 1 & 0 & 0 \\
0 & 0 & 1 & 0
\end{array}\right) \text {. }
$$

Taking $Z=X^{-1}$ and $W=Y^{-1}$, one can show that the 4-tuple $(X, Y, Z, W)$ gives rise to a projective representation $\varrho: G_{L} \rightarrow \mathrm{SU}(3)$ with $[\varrho(x), \varrho(y)]=\omega I=$ $[\varrho(y), \varrho(z)]=[\varrho(z), \varrho(w)]$. The two groups $\operatorname{Stab}(Y)$ and $\operatorname{Stab}(Z)$ act on 4-tuples by

$$
\begin{cases}(X, Y, Z, W) \mapsto\left(X, Y, P Z P^{-1}, P W P^{-1}\right) & \text { for } P \in \operatorname{Stab}(Y), \\ (X, Y, Z, W) \mapsto\left(X, Y, Z, Q W Q^{-1}\right) & \text { for } Q \in \operatorname{Stab}(Z),\end{cases}
$$

and these actions preserve the relations and are nontrivial on conjugacy classes. It follows that the solution set is 6-dimensional and parametrized by $\operatorname{Stab}(Y) / \mathbb{Z}_{4} \times$ $\operatorname{Stab}(Z) / \mathbb{Z}_{4}$, which has Euler characteristic zero. By similar considerations as in the previous case, it follows that $h_{4, a}(L)=0$ for $a=(1,1,1,1)$, and a similar argument shows that $h_{4, a}(L)=0$ for $a=(3,3,3,3)$.

As before, one can perform these computations directly by noting that $L$ is the closure of the braid $\sigma=\sigma_{1}^{2} \sigma_{2}^{2} \sigma_{3}^{2}$ and parametrizing the fixed point set $\operatorname{Fix}(\varepsilon \sigma)$. 
This argument generalizes to the $n$-component chain link in a straightforward manner, as we now explain. The chain link $L$ with $n$ components has link group with presentation

$$
G_{L}=\left\langle x_{1}, \ldots, x_{n} \mid\left[x_{1}, x_{2}\right]=\cdots=\left[x_{n-1}, x_{n}\right]=1\right\rangle .
$$

By Theorem 4.1, up to conjugacy, there is a unique irreducible pair $\left(X_{1}, X_{2}\right) \in$ $\mathrm{SU}(N) \times \mathrm{SU}(N)$ satisfying the equation $\left[X_{1}, X_{2}\right]=\omega I$. A solution is obtained by taking $X_{1}=X$ and $X_{2}=Y$ for $X, Y$ as in the statement of the theorem, and setting $X_{i+2}=X_{i}^{-1}$ for $i=1, \ldots, n-2$, the $n$-tuple

$$
\left(X_{1}, \ldots, X_{n}\right) \in \mathrm{SU}(N) \times \cdots \times \mathrm{SU}(N)
$$

is easily seen to satisfy the relations

$$
\left[X_{1}, X_{2}\right]=\cdots=\left[X_{n-1}, X_{n}\right]=\omega I .
$$

For $i=3, \ldots, n$, the group $\operatorname{Stab}\left(X_{i}\right)$ acts on $n$-tuples by

$$
\left(X_{1}, \ldots, X_{n}\right) \mapsto\left(X_{1}, \ldots, X_{i}, P X_{i+1} P^{-1}, \ldots, P X_{n} P^{-1}\right)
$$

for $P \in \operatorname{Stab}\left(X_{i}\right)$. These actions preserve the relations and are nontrivial on conjugacy classes. Since each $\operatorname{Stab}\left(X_{i}\right) \cong T^{N-1}$ is a maximal torus, it follows that the solution set has dimension $(N-1)(N-2)$ and is parametrized by $\operatorname{Stab}\left(X_{3}\right) / \mathbb{Z}_{N} \times$ $\cdots \times \operatorname{Stab}\left(X_{n}\right) / \mathbb{Z}_{N}$, which has Euler characteristic zero. It follows that $h_{N, a}(L)=0$ for $a=(1, \ldots, 1)$, and a similar argument shows that $h_{N, a}(L)=0$ for $a=(d, \ldots, d)$ for any $d$ relatively prime to $N$.

4B. Split Links. In this section, we consider links $L \subset S^{3}$ that are geometrically split and prove a vanishing result for $h_{N, a}(L)$ provided that the labels satisfy the following condition. Assume $L$ is a link with $n$ components, and suppose it is split. Denoting the components of $L$ by $\ell_{1} \cup \cdots \cup \ell_{n}$, this means that $L=L_{1} \cup L_{2}$, where up to reordering $L_{1}=\ell_{1} \cup \cdots \cup \ell_{n_{1}}$ and $L_{2}=\ell_{n_{1}+1} \cup \cdots \cup \ell_{n}$ are sublinks that are separated by a 2 -sphere. We shall assume that the labels $\left(a_{1}, \ldots, a_{n}\right)$ satisfy the additional condition:

$$
a_{1}+a_{2}+\cdots+a_{n_{1}} \text { is not a multiple of } N .
$$

Using Markov moves we can always find a split braid representative $\beta \in B_{k}$ of $L$; see Figure 3. This means that $\beta=\beta_{1} \beta_{2}$ where

$$
\beta_{1} \in \operatorname{Im}\left(B_{k_{1}} \stackrel{i_{1}}{\hookrightarrow} B_{k}\right) \quad \text { and } \quad \beta_{2} \in \operatorname{Im}\left(B_{k_{2}} \stackrel{i_{2}}{\longrightarrow} B_{k}\right)
$$

for $k=k_{1}+k_{2}$ and $i_{1}, i_{2}$ are injective maps obtained by stabilizing on the right and left, respectively. More precisely, $i_{1}$ takes a braid in $B_{k_{1}}$ and adds $k_{2}$ trivial strands on the right, and $i_{2}$ takes a braid in $B_{k_{2}}$ and adds $k_{1}$ trivial strands on the left. Any 

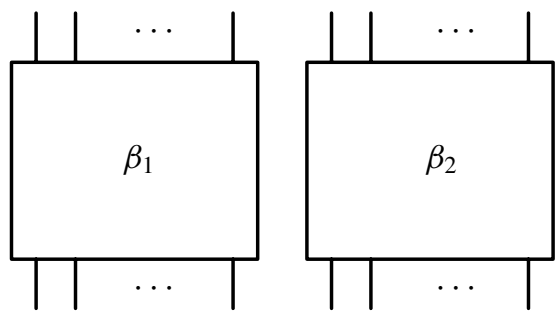

Figure 3. A split braid.

link $L$ obtained as the closure $\hat{\beta}$ of a split braid is obviously a split link, and any split link $L$ can be obtained as the closure of a split braid.

Proposition 4.6. Suppose $L$ is a split link and that $\beta$ is a split braid with $\hat{\beta}=L$. Suppose further that $a=\left(a_{1}, \ldots, a_{n}\right)$ is an n-tuple of labels satisfying (15), and $\varepsilon=\left(\varepsilon_{1}, \ldots, \varepsilon_{k}\right)$ is a compatible $k$-tuple. Then the intersection $\Delta_{k} \cap \Gamma_{\varepsilon \beta}=\varnothing$, and consequently $h_{N, a}(L)=0$.

Proof. Let $X \in \Delta_{k} \cap \Gamma_{\varepsilon \beta}$, then by (8),

$$
X_{1} \cdots X_{k_{1}}=\omega^{d n_{1}} \beta(X)_{1} \cdots \beta(X)_{k_{1}} .
$$

Since $\beta=\beta_{1} \beta_{2}$ is a split braid with $\beta_{1} \in B_{k_{1}}$, by (2) we have that

$$
\beta(X)_{1} \cdots \beta(X)_{k_{1}}=\beta_{1}(X)_{1} \cdots \beta_{1}(X)_{k_{1}}=X_{1} \cdots X_{k_{1}},
$$

and this implies

$$
X_{1} \cdots X_{k_{1}}=\omega^{a_{1}+\cdots+a_{n_{1}}} X_{1} \cdots X_{k_{1}} .
$$

But $\omega^{a_{1}+\cdots+a_{n_{1}}} \neq 1$ by assumption (15), and this gives the desired contradiction.

4C. Concluding remarks. One can give an alternative interpretation of the invariants $h_{N, a}(L)$ in terms of a signed count of conjugacy classes of representations $\varrho: G_{L} \rightarrow \mathrm{PU}(N)$ of the link group as follows. We begin by recalling the classification of principal $\mathrm{PU}(N)$ bundles from [Woodward 1982].

The classifying space $B P U(N)$ is simply connected and has $\pi_{2}(B P U(N))=\mathbb{Z}_{N}$, and an application of the main theorem of [loc. cit.] implies that principal $\mathrm{PU}(N)$ bundles $P \rightarrow X$ over a 3-complex $X$ are determined by the characteristic class $w(P) \in H^{2}\left(X ; \mathbb{Z}_{N}\right)$. In case $N=2, \mathrm{PU}(2)=S O(3)$ and $w(P)$ coincides with the second Stiefel-Whitney class.

Let $L \subset S^{3}$ be a link and $M_{L}=S^{3} \backslash \tau(L)$ its exterior. A projective $\operatorname{SU}(N)$ representation induces a representation $\varrho: G_{L} \rightarrow \mathrm{PU}(N)$, and we denote the associated cohomology class by $w(\varrho) \in H^{2}\left(G_{L} ; \mathbb{Z}_{N}\right)$. The class $w(\varrho)$ vanishes if and only if $\varrho$ lifts to an $\operatorname{SU}(N)$ representation. Further, there is a canonical 
injection $H^{2}\left(G_{L} ; \mathbb{Z}_{N}\right) \rightarrow H^{2}\left(M_{L} ; \mathbb{Z}_{N}\right)$ which is an isomorphism if and only if $M_{L}$ is aspherical, i.e., if and only if $L$ is nonsplit.

By reduction $\bmod N$, an allowable $n$-tuple $\left(a_{1}, \ldots, a_{n}\right) \in \mathbb{Z}^{n}$ determines a unique cohomology class $\bar{w}\left(a_{1}, \ldots, a_{n}\right) \in H^{2}\left(\partial M_{L} ; \mathbb{Z}_{N}\right) \cong\left(\mathbb{Z}_{N}\right)^{n}$. The exact sequence in cohomology for the pair $\left(M_{L}, \partial M_{L}\right)$ gives

$$
\rightarrow H^{2}\left(M_{L} ; \mathbb{Z}_{N}\right) \stackrel{i^{*}}{\longrightarrow} H^{2}\left(\partial M_{L} ; \mathbb{Z}_{N}\right) \rightarrow H^{3}\left(M_{L}, \partial M_{L} ; \mathbb{Z}_{N}\right) \rightarrow 0 ;
$$

and condition (iii) from Section $2 \mathrm{~B}$ guarantees that $\bar{w}\left(a_{1}, \ldots, a_{n}\right)$ lies in the image of $i^{*}$ and hence determines a class $w\left(a_{1}, \ldots, a_{n}\right) \in H^{2}\left(M_{L} ; \mathbb{Z}_{N}\right)$. Condition (ii) implies that the class $w\left(a_{1}, \ldots, a_{n}\right)$ has order $N$.

From this point of view, the invariant $h_{N, a}(L)$ is closely related to the signed count of conjugacy classes of representations $\varrho: G_{L} \rightarrow \mathrm{PU}(N)$ such that $w(\varrho)=$ $w\left(a_{1}, \ldots, a_{n}\right)$. Proposition 4.6 is therefore a direct consequence of the fact that for split links $L$ and allowable $n$-tuples $\left(a_{1}, \ldots, a_{n}\right)$ satisfying condition (15), the associated cohomology class $w\left(a_{1}, \ldots, a_{n}\right)$ does not lie in the image of the map $H^{2}\left(G_{L} ; \mathbb{Z}_{N}\right) \rightarrow H^{2}\left(M_{L} ; \mathbb{Z}_{N}\right)$.

As mentioned in the introduction, it would be interesting to investigate the relationship between the $\mathrm{SU}(N)$ Casson-Lin invariants studied here and the $\mathrm{SU}(N)$ instanton Floer groups constructed by Kronheimer and Mrowka [2011b; 2011a]. It would also be interesting to understand the relationship between the $\mathrm{SU}(N)$ Casson-Lin invariants and classical link invariants. For example, the main result of [Harper and Saveliev 2010] equates the SU(2) Casson-Lin invariant $h_{2}(L)$ of a two component link $L=\ell_{1} \cup \ell_{2}$ with the linking number $\operatorname{lk}\left(\ell_{1}, \ell_{2}\right)$. The following conjecture, if true, would give a generalization to the higher rank setting.

Conjecture 4.7. If $L=\ell_{1} \cup \ell_{2}$ is a two component link in $S^{3}$, then the $\mathrm{SU}(N)$ Casson-Lin invariant satisfies

$$
h_{N, a}(L)=\operatorname{lk}\left(\ell_{1}, \ell_{2}\right)^{N-1} .
$$

This conjecture is consistent with all known computations of the $\mathrm{SU}(N)$ CassonLin invariants, and it's possible that the invariants $h_{N, a}(L)$ are generally invariant under link homotopy. We hope to explore these topics in future work.

\section{Acknowledgements}

Both authors would like to thank Chris Herald, Andy Nicas, and Nikolai Saveliev for their valuable input. We would also like to thank an anonymous referee for many helpful suggestions. Boden expresses his gratitude to the Max Planck Institute for Mathematics in Bonn for its support. 


\section{References}

[Alexander 1923] J. Alexander, "A lemma on systems of knotted curves", Proc. Natl. Acad. Sci. USA 9:3 (1923), 93-95.

[Birman 1974] J. S. Birman, Braids, links, and mapping class groups, Princeton University Press, 1974. MR 0375281

[Boden and Herald 1999] H. U. Boden and C. M. Herald, "A connected sum formula for the SU(3) Casson invariant", J. Differential Geom. 53:3 (1999), 443-464. MR 1806067

[Collin and Steer 1999] O. Collin and B. Steer, "Instanton Floer homology for knots via 3-orbifolds", J. Differential Geom. 51:1 (1999), 149-202. MR 1703606

[Fenn et al. 1997] R. Fenn, R. Rimányi, and C. Rourke, "The braid-permutation group”, Topology 36:1 (1997), 123-135. MR 1410467

[Floer 1988] A. Floer, "An instanton-invariant for 3-manifolds", Comm. Math. Phys. 118:2 (1988), 215-240. MR 956166

[Harper and Saveliev 2010] E. Harper and N. Saveliev, "A Casson-Lin type invariant for links", Pacific J. Math. 248:1 (2010), 139-154. MR 2734168

[Harper and Saveliev 2012] E. Harper and N. Saveliev, "Instanton Floer homology for two-component links”, J. Knot Theory Ramifications 21:5 (2012), 1250054, 8. MR 2902278

[Herald 1997] C. M. Herald, "Flat connections, the Alexander invariant, and Casson's invariant", Comm. Anal. Geom. 5:1 (1997), 93-120. MR 1456309

[Heusener and Kroll 1998] M. Heusener and J. Kroll, "Deforming abelian SU(2)-representations of knot groups”, Comment. Math. Helv. 73:3 (1998), 480-498. MR 1633375

[Kassel and Turaev 2008] C. Kassel and V. Turaev, Braid groups, Graduate Texts in Mathematics 247, Springer, New York, 2008. MR 2435235

[Kronheimer and Mrowka 2011a] P. B. Kronheimer and T. S. Mrowka, "Khovanov homology is an unknot-detector”, Publ. Math. Inst. Hautes Études Sci. 113 (2011), 97-208. MR 2805599

[Kronheimer and Mrowka 2011b] P. B. Kronheimer and T. S. Mrowka, "Knot homology groups from instantons", J. Topol. 4:4 (2011), 835-918. MR 2860345

[Lin 1992] X.-S. Lin, "A knot invariant via representation spaces”, J. Differential Geom. 35:2 (1992), 337-357. MR 1158339

[Long 1989] D. D. Long, "On the linear representation of braid groups", Trans. Amer. Math. Soc. 311:2 (1989), 535-560. MR 943606

[Ruberman and Saveliev 2004] D. Ruberman and N. Saveliev, "Rohlin's invariant and gauge theory, I: Homology 3-tori”, Comment. Math. Helv. 79:3 (2004), 618-646. MR 2081729

[Taubes 1990] C. H. Taubes, "Casson's invariant and gauge theory”, J. Differential Geom. 31:2 (1990), 547-599. MR 1037415

[Woodward 1982] L. M. Woodward, "The classification of principal $\mathrm{PU}_{n}$-bundles over a 4-complex", J. London Math. Soc. (2) 25:3 (1982), 513-524. MR 657508

Received June 11, 2015. Revised March 5, 2016. 
HANS U. BODEN

DEPARTMENT OF MATHEMATICS AND STATISTICS

MCMASTER UNIVERSITY

1280 MAIN ST. W.

HAMILTON ON L8S 4K1

CANADA

boden@mcmaster.ca

ERIC HARPER

4704 Cedar Glen Place

CASTlE Rock, CO 80109

UNITED STATES

ericharper228@gmail.com 


\title{
PACIFIC JOURNAL OF MATHEMATICS
}

Founded in 1951 by E. F. Beckenbach (1906-1982) and F. Wolf (1904-1989)

$$
\text { msp.org/pjm }
$$

\section{EDITORS}

\author{
Don Blasius (Managing Editor) \\ Department of Mathematics \\ University of California \\ Los Angeles, CA 90095-1555 \\ blasius@math.ucla.edu
}

\author{
Paul Balmer \\ Department of Mathematics \\ University of California \\ Los Angeles, CA 90095-1555 \\ balmer@math.ucla.edu \\ Robert Finn \\ Department of Mathematics \\ Stanford University \\ Stanford, CA 94305-2125 \\ finn@math.stanford.edu \\ Sorin Popa \\ Department of Mathematics \\ University of California \\ Los Angeles, CA 90095-1555 \\ popa@math.ucla.edu
}

\author{
Vyjayanthi Chari \\ Department of Mathematics \\ University of California \\ Riverside, CA 92521-0135 \\ chari@math.ucr.edu \\ Kefeng Liu \\ Department of Mathematics \\ University of California \\ Los Angeles, CA 90095-1555 \\ liu@math.ucla.edu \\ Igor Pak \\ Department of Mathematics \\ University of California \\ Los Angeles, CA 90095-1555 \\ pak.pjm@gmail.com \\ Paul Yang \\ Department of Mathematics \\ Princeton University \\ Princeton NJ 08544-1000 \\ yang@math.princeton.edu
}

\section{PRODUCTION}

Silvio Levy, Scientific Editor, production@msp.org

\section{SUPPORTING INSTITUTIONS}

ACADEMIA SINICA, TAIPEI

CALIFORNIA INST. OF TECHNOLOGY

STANFORD UNIVERSITY

UNIV. OF BRITISH COLUMBIA

UNIV. OF CALIFORNIA, BERKELEY

UNIV. OF CALIFORNIA, DAVIS

UNIV. OF CALIFORNIA, LOS ANGELES

UNIV. OF CALIFORNIA, RIVERSIDE

UNIV. OF CALIFORNIA, SAN DIEGO

UNIV. OF CALIF., SANTA BARBARA
KEIO UNIVERSITY

MATH. SCIENCES RESEARCH INSTITUTE

NEW MEXICO STATE UNIV.

OREGON STATE UNIV.
Daryl Cooper

Department of Mathematics

University of California

Santa Barbara, CA 93106-3080 cooper@math.ucsb.edu

Jiang-Hua Lu

Department of Mathematics

The University of Hong Kong

Pokfulam Rd., Hong Kong

jhlu@maths.hku.hk

$$
\text { Jie Qing }
$$

Department of Mathematics

University of California

Santa Cruz, CA 95064

qing@ cats.ucsc.edu

\author{
UNIV. OF CALIF., SANTA CRUZ \\ UNIV. OF MONTANA \\ UNIV. OF OREGON \\ UNIV. OF SOUTHERN CALIFORNIA \\ UNIV. OF UTAH \\ UNIV. OF WASHINGTON \\ WASHINGTON STATE UNIVERSITY
}

These supporting institutions contribute to the cost of publication of this Journal, but they are not owners or publishers and have no responsibility for its contents or policies.

See inside back cover or msp.org/pjm for submission instructions.

The subscription price for 2016 is US $\$ 440 /$ year for the electronic version, and \$600/year for print and electronic.

Subscriptions, requests for back issues and changes of subscriber address should be sent to Pacific Journal of Mathematics, P.O. Box 4163, Berkeley, CA 94704-0163, U.S.A. The Pacific Journal of Mathematics is indexed by Mathematical Reviews, Zentralblatt MATH, PASCAL CNRS Index, Referativnyi Zhurnal, Current Mathematical Publications and Web of Knowledge (Science Citation Index).

The Pacific Journal of Mathematics (ISSN 0030-8730) at the University of California, c/o Department of Mathematics, 798 Evans Hall \#3840, Berkeley, CA 94720-3840, is published twelve times a year. Periodical rate postage paid at Berkeley, CA 94704, and additional mailing offices. POSTMASTER: send address changes to Pacific Journal of Mathematics, P.O. Box 4163, Berkeley, CA 94704-0163.

PJM peer review and production are managed by EditFLOW ${ }^{\circledR}$ from Mathematical Sciences Publishers.

PUBLISHED BY

\section{I. mathematical sciences publishers}

nonprofit scientific publishing

http://msp.org/

(C) 2016 Mathematical Sciences Publishers 


\section{PACIFIC JOURNAL OF MATHEMATICS}

Volume $285 \quad$ No. $2 \quad$ December 2016

The $\mathrm{SU}(N)$ Casson-Lin invariants for links

HANS U. BODEN and ERIC HARPER

The SU(2) Casson-Lin invariant of the Hopf link

HANS U. BODEN and CHRISTOPHER M. HERALD

Commensurations and metric properties of Houghton's groups

José BuRILlo, SEAn Cleary, ARMANDo MaRTino and ClaAs

E. RÖVER

Conformal holonomy equals ambient holonomy

ANDREAS ČAP, A. RoD GOVER, C. RoBIN GRAHAM and

MATTHIAS HAMMERL

Nonorientable Lagrangian cobordisms between Legendrian knots

ORSOLA CAPOVILLA-SEARLE and LISA TRAYNOR

A strong multiplicity one theorem for $\mathrm{SL}_{2}$

JINGSONG CHAI and QING ZHANG

The Yamabe problem on noncompact CR manifolds

PAK TUNG Ho and SeONGTAG KIM

Isometry types of frame bundles

WOUTER VAN LIMBEEK

Bundles of spectra and algebraic K-theory

JOHN A. LIND

Hidden symmetries and commensurability of 2-bridge link complements

CHRISTIAN MiLlichaP and WILLIAM WORDEN

On seaweed subalgebras and meander graphs in type $C$

DMITRI I. PANYUSHEV and OKSANA S. YAKIMOVA

The genus filtration in the smooth concordance group 